\title{
The spiral wind-up of vorticity in an inviscid planar vortex
}

\author{
By ANDREW P. BASSOM AND ANDREW D. GILBERT \\ School of Mathematical Sciences, University of Exeter, North Park Road, \\ Exeter, Devon EX4 4QE, UK \\ email: drew@maths.exeter.ac.uk; adg@maths.exeter.ac.uk
}

(Received 8 October 1997 and in revised form 30 March 1998)

The relaxation of a smooth two-dimensional vortex to axisymmetry, also known as 'axisymmetrization', is studied asymptotically and numerically. The vortex is perturbed at $t=0$ and differential rotation leads to the wind-up of vorticity fluctuations to form a spiral. It is shown that for infinite Reynolds number and in the linear approximation, the vorticity distribution tends to axisymmetry in a weak or coarse-grained sense: when the vorticity field is integrated against a smooth test function the result decays asymptotically as $t^{-\lambda}$ with $\lambda=1+\left(n^{2}+8\right)^{1 / 2}$, where $n$ is the azimuthal wavenumber of the perturbation and $n \geqslant 1$. The far-field stream function of the perturbation decays with the same exponent. To obtain these results the paper develops a complete asymptotic picture of the linear evolution of vorticity fluctuations for large times $t$, which is based on that of Lundgren (1982).

\section{Introduction}

In fluid flow at high Reynolds number there is a tendency for vorticity to aggregate to form coherent vortices, both for planar flows (e.g. McWilliams 1984, 1990; Benzi et al. 1986; Brachet et al. 1988) and in three dimensions (e.g. Kuo \& Corrsin 1971, 1972; Siggia 1981; Kerr 1985; She, Jackson \& Orszag 1990; Vincent \& Meneguzzi 1991). Such concentrations of vorticity are associated with differential rotation of the fluid, both inside and outside the vortex. This causes stretching of fluid elements and means that fluctuations of vorticity (or a passive scalar) are wound up into characteristic spiral structures, and so are driven to small scales. Our goal is to discuss some of the consequences of differential rotation and this winding-up process and its implications for the behaviour of vorticity and scalars in coherent planar vortices.

We consider an idealized problem in which a smooth axisymmetric vortex with vorticity $\Omega=\Omega_{0}(r)$ and associated stream function $\Psi=\Psi_{0}(r)$ is perturbed so as to gain small non-axisymmetric components, and we study their subsequent evolution. If this event occurs at $t=0$ then for $t>0$ the total vorticity and stream function may be written

$$
\Omega=\Omega_{0}(r)+\varepsilon \omega(r, t) \mathrm{e}^{\mathrm{i} n \theta}+\text { c.c. }+O\left(\varepsilon^{2}\right), \quad \Psi=\Psi_{0}(r)+\varepsilon \psi(r, t) \mathrm{e}^{\mathrm{i} n \theta}+\text { c.c. }+O\left(\varepsilon^{2}\right),(1.1 a, b)
$$

where $(r, \theta)$ denote the usual plane polar coordinates, $n \geqslant 1$ and $\varepsilon \ll 1$. Here $\omega$ and $\psi$ are generally complex and 'c.c.' refers to the complex conjugate of the preceding expression. Our study will apply to a wide class of perturbations, but as an important example (Lingevitch \& Bernoff 1995, henceforth referred to as LB95) we can imagine 


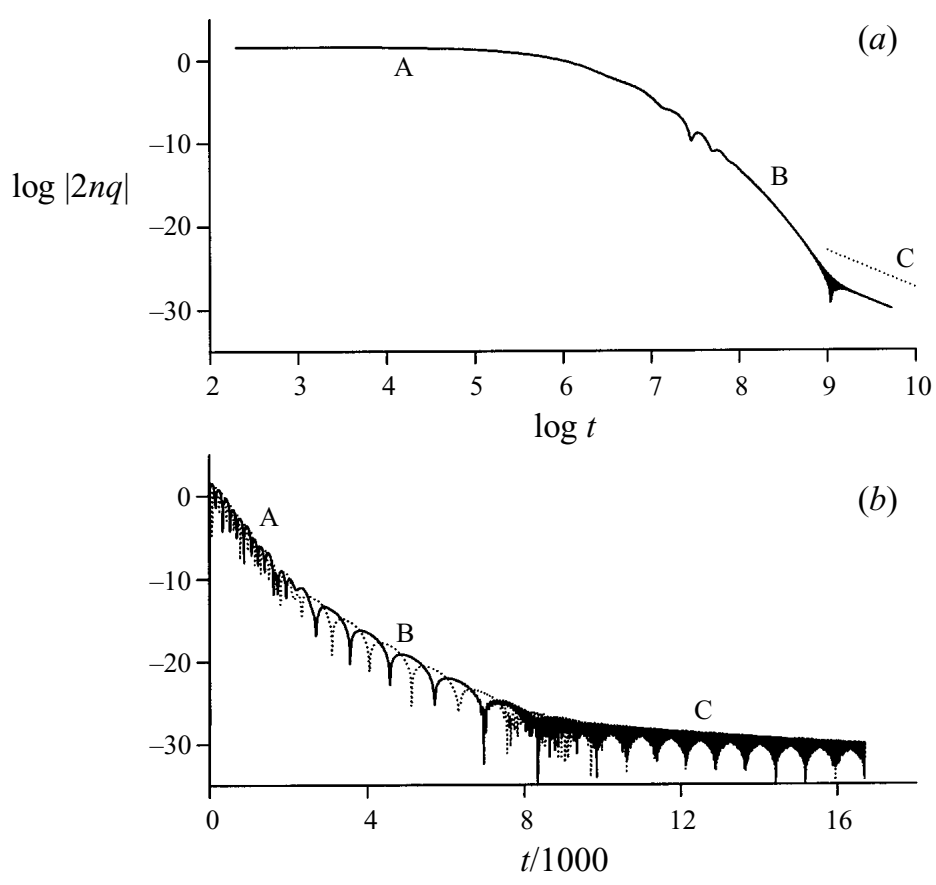

Figure 1. (a) $\log |2 n q(t)|$ (solid) plotted against $\log t$ for the vortex (1.3) with $n=2, L=\gamma=1$. The three regimes labelled (A)-(C) are described in the text and the dotted line has slope -4.464 . (b) $\log |\operatorname{Re} 2 n q(t)|$ (solid) and $\log |\operatorname{Im} 2 n q(t)|$ (dotted) plotted against scaled time $t / 1000$.

briefly and impulsively imposing a small external irrotational flow at $t=0$. This perturbation creates a non-axisymmetric component of vorticity, and is equivalent to imposing a certain initial condition at $t=0^{+}$. For $t>0$ the non-axisymmetric part of the distribution is subject to differential rotation and the vorticity becomes wound into a spiral.

We study this process within linear theory, i.e. only to order $\varepsilon$, and at infinite Reynolds number, Re. The first problem of interest is the evolution of the far field of the vortex. At large distances the perturbation stream function will be irrotational and so behaves as

$$
\psi(r, t) \sim q(t) r^{-n} \quad(r \rightarrow \infty) .
$$

What can be deduced about the behaviour of $q(t)$ and, in particular, what is its inviscid asymptotic behaviour for large time $t$ ?

To indicate why we find this question intriguing we show in figure $1(a)$ a plot of $\log |2 n q(t)|$ against $\log t$ obtained numerically for a vortex with a Gaussian distribution of vorticity (Lamb 1932, §334a). (The factor $2 n$ is for convenience; see equation (2.22) below.) This vortex takes the form

$$
\Omega_{0}(r)=\frac{\gamma}{4 \pi L^{2}} \exp \left(-r^{2} / 4 L^{2}\right), \quad \Psi_{0}(r)=-\frac{\gamma}{2 \pi} \int_{0}^{r}\left[1-\exp \left(-r^{2} / 4 L^{2}\right)\right] \frac{\mathrm{d} r}{r},
$$

having length scale $L$ and total circulation $\gamma$. Figure 1(a) illustrates the evolution of $q(t)$ for the particular choices $n=2, L=1$ and $\gamma=1$ and the result is a somewhat confusing curve with some strange kinks and a final power law like $t^{-\lambda}$ with $\lambda \simeq 4.5$. There are in fact three distinct regimes here: two transients labelled (A) and (B), followed by the asymptotic $t^{-\lambda}$ power law (C). The different regimes 
are seen more clearly in figure $1(b)$ which shows the phase behaviour of $q(t)$ by plotting $\log |\operatorname{Re} 2 n q(t)|$ (solid) and $\log |\operatorname{Im} 2 n q(t)|$ (dotted) against $t / 1000$ (rather than $\log t$ ). The downward spikes correspond to zero crossings. It may be seen that the three regimes correspond to different frequency behaviour, and that the kinks mark a transition from regime (A) to (B). The primary objective in this work is to understand these various regimes and especially the origin of the power law in $(\mathrm{C})$ and the value of $\lambda$.

A second aim is to quantify and understand the inviscid relaxation of the vortex to axisymmetry. This process of 'axisymmetrization' has been observed widely in twodimensional turbulence and contour dynamics simulations (e.g. Melander, McWilliams \& Zabusky 1987; Yao \& Zabusky 1996; Rossi, Lingevitch \& Bernoff 1997) and more generally in geophysical fluid dynamics (e.g. McCalpin 1987; Sutyrin 1989; Smith \& Montgomery 1995). Fluctuations that are generated tend to wind up into a spiral structure because of the differential rotation of the vortex. As time increases the spiral becomes ever tighter and the vortex becomes axisymmetric. However, this is not a process in which the vorticity tends pointwise to an axisymmetric distribution; indeed there remain vorticity fluctuations of order unity indefinitely, in the absence of viscosity. Rather it is that the fluctuations are driven to ever smaller scales, and so the vorticity only becomes axisymmetric in a coarse-grained or average sense. To quantify this process we study the evolution of vorticity in a 'weak' sense. This is defined precisely below, but the idea is that we take a fixed smooth test function $f$ and look at the evolution of the inner product $(f, \omega)$, i.e. the integral of $f^{*} \omega$ over space. This taking of the inner product provides a suitable averaging process and it is found that $(f, \omega)$ decays, with an asymptotic power law again of $t^{-\lambda}$ for typical test functions $f$. The exponent $\lambda$ depends only on the harmonic $n$ and is independent of the underlying vortex provided that it has a 'generic' structure, obeying a set of natural conditions given in $\S 2$ below.

In an average sense then the non-axisymmetric vorticity distribution $\omega$ tends to zero, and so the original axisymmetric vortex is linearly 'asymptotically stable' in this weak sense even for $R e=\infty$. This complements the result of Bernoff \& Lingevitch (1994, hereafter referred to as BL94) that for finite $R e$ a vortex is asymptotically stable in the sense of pointwise convergence to axisymmetry (discussed further below). It is also known that such a vortex is nonlinearly Liapunov stable for $R e=\infty$ (Saffman 1985, 1992; Wan \& Pulvirenti 1985; Dritschel 1988a). The question of the weak convergence of $\omega$ as $t \rightarrow \infty$ is closely related to the evolution of $q(t)$ discussed above: the decay of the far field with time is just one measure of the relaxation of the vortex to axisymmetry. The focus on weak measures of convergence appears a useful way to discuss the creation of fine structure in systems such as this, and has found application in broadly related magnetic dynamo problems (see e.g. Childress \& Gilbert 1995).

To understand these weak decay processes, it is necessary to obtain a complete asymptotic description of the winding-up of vorticity, valid for all space in the limit $t \rightarrow \infty$, and this is our third and final aim. Our starting point is Lundgren's (1982) asymptotic framework; here we are referring to the analysis of planar vortices in his appendix A, rather than the transformation to three-dimensional axially strained vortices, which is the main focus of that paper. The key idea is that as the vorticity $\omega$ winds up and goes to smaller scales, the stream function decays for geometrical reasons and, at late times, the vorticity behaves as a passive scalar in the flow field given by $\Omega_{0}$, with the stream function giving only small corrections. While this asymptotic analysis captures many of the essential features of the winding-up of $\omega$ and decay of $\psi$, it does not throw any light on the behaviour of $q(t)$. The expansion 
also becomes non-uniform at the points where differential rotation is small (as noted by Lundgren) and this occurs at the origin of the vortex, in a region $r=O\left(t^{-1 / 2}\right)$. Although this is a small and shrinking region of space, because $\psi$ is determined non-locally from $\omega$, the region exerts an influence over the flow throughout space and, perhaps surprisingly, determines the far-field behaviour of $\psi$ and so $q(t)$.

Our analytical study is strictly inviscid, and although we use low levels of viscosity in our numerical calculations, it is not significant in any of the results we show. The problem of the evolution of perturbations to an axisymmetric vortex at high finite $R e$ is considered by BL94. The emphasis of that work is on the interaction of viscosity and the generation of small scales by shear to eliminate non-axisymmetric vorticity. This is titled a 'shear-diffuse mechanism' and operates on a rapid time scale of order $R e^{1 / 3}$, leading to the asymptotic stability of axisymmetric vortices, in a strong sense: at each point in space the vorticity tends to an axisymmetric distribution at large time. This is also implicit in Lundgren's (1982) paper, and has been discussed for a passive scalar or vorticity by Rhines \& Young $(1982,1983)$, Moffatt \& Kamkar (1983) and Gilbert (1988). Of course there are close parallels with our study: in BL94 the generation of fine scales by shear enhances viscosity and leads to rapid viscous decay and axisymmetry, while in our work it is the same creation of small scales that leads to the decay of weak measures of the vorticity, and so to axisymmetry in a coarse-grained sense. In our paper the emphasis is on $R e=\infty$ and stability in this weak sense, which means we have to probe the asymptotic structure of the flow more deeply than was done in BL94.

Nevertheless there is one key idea that we take from their work with only minor modification. They introduce and provide evidence for a

MIXING HYPOTHESIS (BL94): If an axisymmetric vorticity distribution is subject to a linear non-axisymmetric perturbation that preserves the first moment of vorticity, this perturbation will decay on a time scale of order $\mathrm{Re}^{1 / 3}$.

The condition that the first moment

$$
(\bar{x}, \bar{y})=\gamma^{-1} \int(x, y) \Omega \mathrm{d}^{2} \boldsymbol{r} \quad\left(\gamma=\int \Omega \mathrm{d}^{2} \boldsymbol{r}\right),
$$

is zero is to factor out solid body translations of the vortex, which cannot decay in time. BL94 also impose conditions on the class of vortices to which this hypothesis applies, and these amount to conditions (2.15) below. For $R e=\infty$ pointwise decay of vorticity cannot be expected, and so our version of this hypothesis is

MIXING HYPOTHESIS FOR $R e=\infty$ : If an axisymmetric vorticity distribution is subject to a linear non-axisymmetric perturbation that preserves the first moment of vorticity, this perturbation will decay weakly to zero on the turnover time scale.

Note that the turnover time scale is the only one left in the problem for $R e=\infty$. We are not in a position to prove either hypothesis, although our numerical simulations offer support for the latter. Instead we assume the $R e=\infty$ hypothesis, and build an asymptotic framework to describe the vorticity as $t \rightarrow \infty$ and its weak decay. Given this hypothesis, our results describe the evolution of a wide class of perturbations to an extensive family of vortices.

The remainder of this paper is structured as follows. The vorticity problem is set up in $\S 2$ and the notion of weak convergence discussed. Both for insight and as a convenient point of comparison we first set up an analogous scalar problem, the development of a passive scalar in a given flow field. This scalar problem is studied 
analytically in $\S 3$ and numerical simulations are presented in $\S 4$. These simulations not only verify the analytical results, but the program proved a useful precursor for that used for the vorticity problem. Some counterparts to the vorticity results shown in figure 1 are obtained in $\S 4$ : these results bear many similarities with figure 1 although there are also some significant differences. Guided by these results, we return in $\S 5$ to the study of vorticity and begin by developing Lundgren's (1982) solution valid for radial distances $r=O(1)$ and as time $t \rightarrow \infty$. This solution is non-uniform at $r^{2} t=O(1)$ and so an inner region is identified which must be analysed separately in order to provide a complete asymptotic picture of the vorticity at large times $t$. An important outcome of our study is that Lundgren's solution is not the only one which is valid for $r=O(1)$ and $t \rightarrow \infty$. We identify a second possible form, which we term the 'Helmholtz solution'; this also becomes invalid when $r=O\left(t^{-1 / 2}\right)$.

The detailed study of the inner region is tackled in $\S 6$ and we find that the vorticity here is governed at leading order by a third-order ordinary differential system subject to suitable matching conditions. Although the analysis of this system is somewhat elaborate, analytical solutions may be obtained in terms of standard Kummer functions. The most significant outcome of this work is that the vorticity distribution tends to axisymmetry in the weak sense described above: furthermore when the vorticity field is integrated against a smooth test function the result decays as $t^{-\lambda}$ with $\lambda=1+\left(n^{2}+8\right)^{1 / 2}$, where $n$ is the azimuthal wavenumber of the perturbation. This explains the origin of the power law decay of regime $(C)$ shown in figure 1 and our findings are supported by numerical simulations, which are described in $\S 7$. In $\S 8$ we give some concluding remarks, and some important but technical issues are relegated to an Appendix.

\section{Formulation of the vorticity problem}

The equations for a planar vortex in a prescribed external irrotational flow may be written

$$
\begin{gathered}
\partial_{t} \Omega=J\left(\Psi+\Psi_{\mathrm{ext}}, \Omega\right)+R e^{-1} \nabla^{2} \Omega, \\
\nabla^{2} \Psi=-\Omega, \quad \nabla^{2} \Psi_{\mathrm{ext}}=0, \\
\nabla^{2} \equiv \partial_{r}^{2}+r^{-1} \partial_{r}+r^{-2} \partial_{\theta}^{2}, \quad J(a, b) \equiv r^{-1}\left(\partial_{r} a \partial_{\theta} b-\partial_{\theta} a \partial_{r} b\right) .
\end{gathered}
$$

In these equations $\Omega$ denotes the vorticity; $\Psi$ is the corresponding stream function determined by inverting (2.2a) and is permitted to grow no faster than $\log r$ for large $r$. The externally imposed irrotational flow is given by the harmonic function $\Psi_{\text {ext }}$ which may increase as a power of $r$. This external flow can be imagined as being imposed by the motion of distant boundaries, paddles or vortices; however it must not introduce vorticity into the region of interest.

We start with an axisymmetric vortex $\Omega=\Omega_{0}(r), \Psi=\Psi_{0}(r)$ for $t<0$. Without loss of generality the non-dimensionalization implicit within equation (2.1) may be taken to be such that the total circulation $\gamma$ and length scale $L$ of the vortex are both unity. At $t=0$ the vortex is supposed to be perturbed instantaneously so as to generate small non-axisymmetric components $\omega$ and $\psi$ as in the expansions (1.1) by, for example, switching $\Psi_{\text {ext }}$ on and off briefly as discussed below. The subsequent free evolution of $\psi$ and $\omega$ at order $\varepsilon$ is given by linearizing equations (2.1), (2.2a) about 
the basic vortex distribution $\left(\Psi_{0}, \Omega_{0}\right)$ and taking $\Psi_{\text {ext }}=0$ to yield

$$
\partial_{t} \omega+\mathrm{in} \alpha(r) \omega+\mathrm{in} \beta(r) \psi=R e^{-1} \Delta \omega
$$

and

$$
\Delta \psi=-\omega \quad\left(\Delta \equiv \partial_{r}^{2}+r^{-1} \partial_{r}-r^{-2} n^{2}\right) .
$$

The angular velocity $\alpha(r)$ and the quantity $\beta(r)$ are given by

$$
\alpha(r)=-r^{-1} \partial_{r} \Psi_{0}, \quad \beta(r)=r^{-1} \partial_{r} \Omega_{0}
$$

and we note that $\Omega_{0}=-r^{-1} \partial_{r}\left(r \partial_{r} \Psi_{0}\right)$. The generation of vorticity from the axisymmetric vorticity $\Omega_{0}$ is contained in the term $\operatorname{in} \beta(r) \psi$ and for the vortex (1.3) (with $L=\gamma=1)$

$$
\alpha(r)=\left(2 \pi r^{2}\right)^{-1}\left[1-\exp \left(-r^{2} / 4\right)\right], \quad \beta(r)=-(8 \pi)^{-1} \exp \left(-r^{2} / 4\right) .
$$

In the remainder of this work frequent reference will be made to the Gaussian vortex (1.3), so that we can apply our results to a concrete example. However, we emphasize that our findings have wider application.

A natural way to generate a non-axisymmetric perturbation to the vortex at $t=0$ is to apply the external irrotational flow $\Psi_{\text {ext }}$ impulsively, with

$$
\Psi_{\text {ext }}=\varepsilon \delta(t) r^{n} \mathrm{e}^{\mathrm{i} n \theta}+\text { c.c. }
$$

in which $\delta(t)$ denotes the Dirac delta function. This disturbance is equivalent to imposing the initial condition on the vortex at time $t=0^{+}$:

$$
\omega\left(r, 0^{+}\right)=-\mathrm{i} n r^{n} \beta(r), \quad \Delta \psi\left(r, 0^{+}\right)=-\omega\left(r, 0^{+}\right),
$$

with the vortex then evolving freely for $t>0$. Equations (2.4)-(2.6) and (2.9) represent the linear initial value problem that is studied in this paper and, except for numerical simulations, $R e$ will be taken to be infinity. In this case there are no free parameters left in the problem once the structure of the basic vortex is fixed by specification of $\alpha(r)$ and $\beta(r)$ (by (2.7) for example).

Switching $\Psi_{\text {ext }}$ on impulsively in this way is a convenient way of generating an initial condition for the vorticity fluctuations, and will play no further role in our study, which will apply to a broad class of initial perturbations subject only to the mixing hypothesis for $R e=\infty$ discussed in $\S 1$. Note however that this initial value problem can be thought of as giving the Green's function for the linear response to external irrotational flows: the response to a continuous irrotational forcing would be given by a convolution integral in the normal way. Thus the initial condition chosen is special but nonetheless natural. This connection with the Green's function has been stressed in LB95 and the function studied for $R e<\infty$.

We are assuming that perturbations to the vortex satisfy the condition of the mixing hypothesis for $R e=\infty$ stated in the introduction. Condition (1.4) is automatically satisfied for $n \geqslant 2$, but for $n=1$ it means that the initial perturbation must obey

$$
\int_{0}^{\infty} \omega(r) r^{2} \mathrm{~d} r=0 \quad(n=1)
$$

which ensures that the initial condition is orthogonal to the time-invariant translation mode of the vortex (see BL94 for discussion of this point). For $n=1$ the stream function (2.8) corresponds to uniform flow and in this case the initial condition (2.9) is an infinitesimal translation which is precisely the time-independent solution that condition (2.10) is designed to exclude. Thus (2.9) cannot be used as a starting 
condition when $n=1$ and so we restrict its use to $n \geqslant 2$. Particular attention will be paid to the case when $n=2$ as this is the lowest value for which irrotational disturbances lead to spiral wind-up. However, by the mixing hypothesis our asymptotic results do hold for $n=1$ perturbations satisfying (2.10) and this is verified numerically in $\S 7$.

Crucial in our analysis is the structure of the basic vortex, defined by $\Omega_{0}$ and $\Psi_{0}$, in regions where the differential rotation $\alpha^{\prime}(r)=\partial_{r} \alpha(r)$ is smallest. We therefore need to consider carefully its behaviour as $r \rightarrow 0$ and $r \rightarrow \infty$ and this leads to a set of conditions which characterize the family of vortices to which our analysis applies. These conditions are natural, define a class of 'generic' vortices, and are of course satisfied by the Gaussian vortex (1.3).

The first requirement is that all physical quantities describing the flow field should be 'smooth': that is, infinitely differentiable throughout space and in particular at the origin. This means that if the Fourier component $a(r) \mathrm{e}^{\mathrm{i} m \theta}$ of a physical quantity is extracted, and the function $a(r)$ expanded in a power series in $r$ for small $r$, this power series must take the form

$$
a(r) \mathrm{e}^{\mathrm{i} m \theta}=r^{m}\left(a_{0}+r^{2} a_{1}+r^{4} a_{2}+\cdots\right) \mathrm{e}^{\mathrm{i} m \theta} \quad(m \geqslant 0) .
$$

That this is a necessary condition is seen by noting that $\nabla^{2} r^{p} \mathrm{e}^{\mathrm{i} m \theta}=\left(p^{2}-m^{2}\right) r^{p-2} \mathrm{e}^{\mathrm{i} m \theta}$ : if a power other than those in (2.11) occurs in the series one could repeatedly apply $\nabla^{2}$ and thereby obtain a non-zero term in a negative power of $r$, contradicting the requirement of being infinitely differentiable at the origin.

This smoothness condition applies to the axisymmetric stream function $\Psi_{0}$ and vorticity $\Omega_{0}$ which expand near the origin in a series of the form (2.11) with $m=0$. From the definitions of $\alpha$ and $\beta$ in (2.7) this leads to similar expansions

$$
\alpha(r)=\alpha_{0}+\alpha_{1} r^{2}+\alpha_{2} r^{4}+\cdots, \quad \beta(r)=\beta_{0}+\beta_{1} r^{2}+\beta_{2} r^{4}+\cdots
$$

and, since $\beta=r^{-1} \partial_{r}\left(r^{-1} \partial_{r}\left[r^{2} \alpha\right]\right)$ from (2.6), the coefficients are related by

$$
\beta_{0}=8 \alpha_{1}, \quad \beta_{1}=24 \alpha_{2}, \quad \ldots .
$$

For the vortex (1.3) (with $\gamma=L=1$ )

$$
\alpha_{0}=1 / 8 \pi, \quad \alpha_{1}=-1 / 64 \pi=\beta_{0} / 8 .
$$

Our next constraint on the basic vortex is that the differential rotation $\alpha^{\prime}(r)$ satisfies

$$
\alpha^{\prime}(r) \neq 0 \quad \text { for } \quad r>0 .
$$

This quantity necessarily vanishes at the origin (see (2.12a)), but we insist that it does not vanish too quickly and so impose

$$
\alpha_{1} \neq 0
$$

this is simply the requirement that the angular velocity not be especially flat at the origin. Also since the vortex has total circulation of unity in our non-dimensionalization,

$$
\alpha(r) \sim 1 / 2 \pi r^{2}, \quad \alpha^{\prime}(r) \sim-1 / \pi r^{3} \quad \text { as } \quad r \rightarrow \infty .
$$

Finally we require that the basic vortex is 'localized', meaning here that the field $\Omega_{0}(r)$ should fall off faster than any power of $r$ as $r \rightarrow \infty$. The perturbation vorticity $\omega(r, t)$ is also taken to be localized at $t=0$ and the reasonable assumption is made that this continues to hold at later times.

In order to measure the weak behaviour of the vorticity field we introduce the 
inner product

$$
(a, b)=\frac{1}{2 \pi} \int_{0}^{2 \pi} \int_{0}^{\infty} a^{*} b r \mathrm{~d} r \mathrm{~d} \theta,
$$

along with a family of test functions $f(r, \theta)$. These are taken to be smooth in space and localized in the sense described above. Since we focus on the behaviour of the perturbation vorticity $\omega(r) \mathrm{e}^{\mathrm{i} n \theta}$ (with $n \geqslant 1$ ), it suffices to take a test function of the same form, $f(r) \mathrm{e}^{\mathrm{i} n \theta}$. The requirement that such a function be smooth means that near the origin $f(r)$ must expand according to

$$
f(r)=r^{n}\left(f_{0}+f_{1} r^{2}+f_{2} r^{4}+\cdots\right) ;
$$

here use has been made of (2.11) with $m=n$. In the remainder of this work we will use the family of test functions

$$
f(r)=r^{n} \exp \left(-r^{2} / 4 l^{2}\right) \quad(l<\infty),
$$

parameterized by the length scale $l$.

From now on we abbreviate the cumbersome expression $\left(f \mathrm{e}^{\mathrm{i} n \theta}, \omega \mathrm{e}^{\mathrm{i} n \theta}\right)$ to

$$
(f, \omega)=\int_{0}^{\infty} f^{*}(r) \omega(r, t) r \mathrm{~d} r
$$

with little risk of confusion. Taking an inner product of this form represents a coarsegraining or local averaging of the vorticity field, and its decay with time quantifies the relaxation of the perturbation vorticity to zero and the vortex to axisymmetry. It may be checked that for test functions

$$
-(f, \omega)=(f, \Delta \psi)=(\Delta f, \psi)
$$

using integration by parts.

One useful function that falls outside our family of test functions is $r^{n}$; although it is the limiting case $l=\infty$ of (2.19) it is not localized. Following integration by parts as used to derive (2.21) leads to the identity

$$
\left(r^{n}, \omega\right)=-\lim _{r \rightarrow \infty} r^{2 n+1} \partial_{r}\left(r^{-n} \psi\right)=2 n q(t) ;
$$

we recall that $q(t)$ is defined in (1.2) and gives the far-field behaviour of $\psi$. This link between $2 n q(t)$ and the inner product $\left(r^{n}, \omega\right)$ is the reason we base our plots on the quantity $2 n q(t)$ rather than $q(t)$.

\section{Analysis of passive scalar wind-up}

Before tackling the vorticity problem as summarized by (2.4)-(2.6), (2.9), it is helpful to examine the analogous but rather easier question of the wind-up and weak decay of a passive scalar $\sigma(r) \mathrm{e}^{\mathrm{i} n \theta}$ in the flow field determined by $\Psi_{0}(r)$. The scalar obeys

$$
\partial_{t} \sigma+\mathrm{in} \alpha(r) \sigma=P^{-1} \Delta \sigma,
$$

which should be compared with (2.4), where $P$ denotes a Péclet number. For our analysis it is assumed that $P=\infty$ and $n \geqslant 1$ while for numerical simulation and comparison with the vorticity problem we use the initial condition analogous to $(2.9 a)$

$$
\sigma\left(r, 0^{+}\right)=-\mathrm{i} n r^{n} \beta(r)
$$


although this form has no particular significance for the passive scalar. With these assumptions the exact solution is

$$
\sigma(r, t)=g(r) \mathrm{e}^{-\mathrm{i} n \alpha(r) t}, \quad g(r)=\sigma\left(r, 0^{+}\right),
$$

which represents the winding-up of the initial condition. At large times, it is plain that $\sigma$ does not decay pointwise although it does vary ever more rapidly as a function of radius $r$, and so should decay weakly. To quantify this behaviour we take a test function $f(r) \mathrm{e}^{\mathrm{i} n \theta}$ and consider

$$
(f, \sigma)=\int_{0}^{\infty} f^{*}(r) g(r) \mathrm{e}^{-\mathrm{i} n \alpha(r) t} r \mathrm{~d} r
$$

as $t \rightarrow \infty$. This is a generalized Fourier integral and can be evaluated asymptotically by standard methods (e.g. Erdélyi 1956, §2.9). It may be shown that

$$
(f, \sigma)=O\left(t^{-n-1}\right)
$$

and so in a weak sense the scalar distribution does indeed converge to zero. Although it is not difficult to verify this result, we give the argument here as it highlights the role of the assumptions made in $\S 2$ above. It is convenient to set $H_{0}(r)=r f^{*}(r) g(r)$ and write (3.4) as

$$
I_{0}=\int_{0}^{\infty} H_{0}(r) \mathrm{e}^{-\mathrm{in} \alpha(r) t} \mathrm{~d} r .
$$

Now $H_{0}=O\left(r^{2 n+1}\right)$ as $r \rightarrow 0$, which follows from (2.11) since $f \mathrm{e}^{\mathrm{i} n \theta}$ and $g \mathrm{e}^{\mathrm{i} n \theta}$ are smooth at the origin with $f \sim f_{0} r^{n}, g \sim g_{0} r^{n}$. We take

$$
H_{0}(r) \sim r^{2 m+1}\left(H_{00}+H_{01} r^{2}+\cdots\right), \quad H_{00}=f_{0}^{*} g_{0}
$$

near the origin and we have set $m=n$ in anticipation of an inductive step that reduces the power $r^{2 m+1}$ of the integrand at the origin to $r^{2 m-1}$. The integral is rewritten as

$$
I_{0}=t^{-1} \int_{0}^{\infty} \frac{H_{0}(r)}{-\mathrm{i} n \alpha^{\prime}}\left\{-\mathrm{i} n \alpha^{\prime} t \mathrm{e}^{-\mathrm{i} n \alpha(r) t}\right\} \mathrm{d} r
$$

and we remember that $\alpha^{\prime}$ vanishes nowhere except at $r=0$ where it is strictly of order $r$ from (2.15). Also $\alpha^{\prime}$ decays algebraically as $r \rightarrow \infty(2.16 b)$ while $H_{0}$ tends to zero faster than any power of $r$. It follows that the quantity $H_{0} /\left(-\mathrm{i} n \alpha^{\prime}\right)$ is finite at the origin, tends to zero rapidly as $r \rightarrow \infty$ and is bounded. Integrating $\{\cdot\}$ in (3.8) using integration by parts and evaluating the boundary term yields

$$
I_{0}= \begin{cases}t^{-1} I_{1} & (m \geqslant 1) \\ t^{-1} I_{1}+t^{-1} H_{00} \mathrm{e}^{-\mathrm{inn} \alpha_{0} t} / 2 i n \alpha_{1} & (m=0)\end{cases}
$$

where

$$
I_{1}=\int_{0}^{\infty} H_{1}(r) \mathrm{e}^{-\mathrm{in} \alpha(r) t} \mathrm{~d} r
$$

and

$$
H_{1}(r)=\partial_{r}\left(\frac{H_{0}}{i n \alpha^{\prime}}\right) \sim \frac{r^{2 m-1}}{i n \alpha_{1}}\left(m H_{00}+(m+1)\left(H_{01}-2 H_{00} \frac{\alpha_{2}}{\alpha_{1}}\right) r^{2}+\cdots\right)
$$

as $r \rightarrow 0$.

Therefore, after starting with the integral $I_{0}$ in which $H_{0}=O\left(r^{2 m+1}\right)$, integration by parts has led to an integral $I_{1}$ of similar type with $H_{1}=O\left(r^{2 m-1}\right)$ together with a 
boundary term if $m=0$. Plainly this procedure can be used repeatedly to reduce $m$ from $n$ in (3.7) right down to zero and then generate a sequence of boundary terms from (3.9) which represents an asymptotic series for the original integral in inverse powers of $t$. Applied to (3.4) this yields the leading term

$$
(f, \sigma)=\frac{n !}{2}\left(\frac{1}{\mathrm{i} n \alpha_{1} t}\right)^{n+1} f_{0}^{*} g_{0} \mathrm{e}^{-\mathrm{in} n \alpha_{0} t}+O\left(t^{-n-2}\right),
$$

which confirms the result (3.5) concerning the weak convergence of the scalar field.

We have given results for the generic case in which the initial scalar field $\sigma\left(r, 0^{+}\right)=$ $g(r)$ has behaviour near the origin $g(r) \sim g_{0} r^{n}$ with $g_{0} \neq 0$, and similarly for the test function $f(r)$. We point out the role of assumption $(2.15 a)$ in ensuring that all the contributions to the integral come from the origin, and (2.15b) in determining the leading asymptotic form. However if one of $f$ or $g$ vanishes as $o\left(r^{n}\right)$ near the origin then the decay of $(f, \sigma)$ will occur at a faster rate, depending on the leading asymptotic form of these functions. If $f(r)$ or $g(r)$ vanishes completely in a neighbourhood of the origin then the convergence will be faster than algebraic and will arise from the rapid oscillations away from the origin; the form of the decay is likely to be quite sensitive to the precise form of $f$ and $g$ in this case, and we have not tried to obtain general results.

\section{Numerical study of scalar wind-up and transient behaviour}

The predictions of $\S 3$ were checked numerically by solving the system (3.1), (3.2) subject to the Gaussian vortex distribution (2.7). The code integrated the partial differential equation (3.1) with mode number $n=2$ and the Péclet number $P=10^{8}$ using $N=6001$ points spread uniformly between $r=0$ and $r=r_{\max }=12$. It might seem more sensible to evaluate the integral (3.4) directly but we chose to solve the PDE as the program was developed partially as a prototype for the vorticity code used and discussed below in $\S 7$.

Figure 2(a) shows $\alpha(r), \alpha^{\prime}(r)$ and $\beta(r)$ for the basic axisymmetric flow field (2.7); note that $\alpha^{\prime}(r)$ vanishes only at $r=0$, as stipulated. Figure $2(b)$ illustrates the resulting scalar distribution $\sigma$ as a function of $r$ at $t=3000$. To test the weak convergence result (3.12), figure $3(a)$ shows $\log |(f, \sigma)|$ (solid) as a function of $\log t$ for the test function of the type (2.19) with the parameter $l=1 / 2$. Clear power law scaling is seen with the correct power law of $t^{-3}$; in fact for $\log t \geqslant 5$ it is largely indistinguishable from the asymptotic leading term (3.12) which is depicted by the dotted line. Note that the range $0 \leqslant t \leqslant 10^{4}$ of $t$ used in the figure at first sight appears rather large (also see figure 1). This is because even though the vortex has circulation and scale of unity, the decay is governed by the combination $n \alpha_{1} t$ (see (3.12)) and the coefficient $\alpha_{1}$, although strictly $O(1)$, is numerically small for the Gaussian vortex, with $\alpha_{1}=-1 / 64 \pi \simeq-1 / 200$ from $(2.14 b)$.

The value $l=1 / 2$ used in figure 3(a) shows most clearly the power law scaling. For larger values of $l$, the asymptotic power law eventually emerges after a transient, which can be long. To illustrate this, and for comparison with the vorticity problem (see figure $1 a$ ), in figure $3(b)$ we plot $\log |(f, \sigma)|$ for the limiting case $l=\infty$, that is $f(r)=r^{2}$. The transient occurs for $\log t \leqslant 8$, after which the correct power law emerges. A hint as to the cause of the transient may be seen from the scalar distribution shown in figure 2(b); the asymptotic result (3.12) arises from the region of slow oscillations near the origin; by 'slow' we mean oscillations varying relatively slowly in space compared 

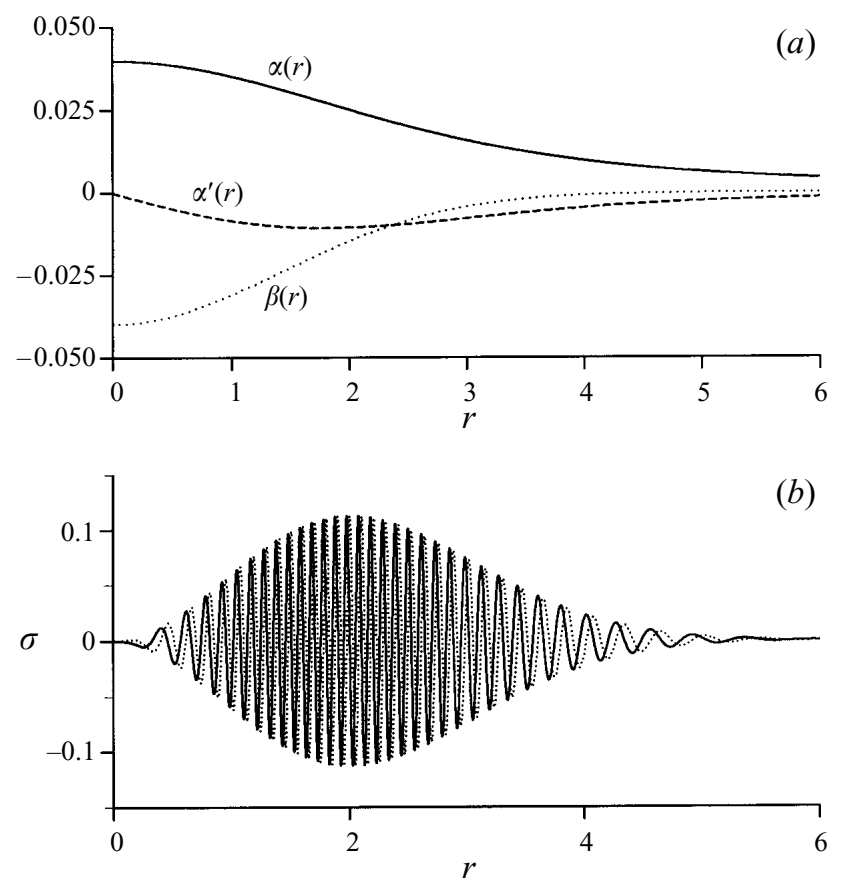

Figure 2. (a) The flow structure: $\alpha(r)$ (solid), $\alpha^{\prime}(r)$ (dashed) and $\beta(r)$ (dotted) as given by (2.7).

(b) Scalar distribution $\operatorname{Re} \sigma$ (solid) and $\operatorname{Im} \sigma$ (dashed) against $r$, at $t=3000$ for $n=2$.

with the rapid oscillations for $r=O(1)$. However there is also a zone of slow variation for moderately large $r$ around $r \simeq 4.5$ and this is the source of the transient, which is of course subdominant to (3.12) for large time $t$.

To analyse this transient we consider the contribution from large $r$ to the integral $(f, \sigma)$ (cf. BL94, § III). Substituting $f(2.19)$ and $\sigma(2.7),(3.2),(3.3)$ in $(f, \sigma)$ yields

$$
(f, \sigma)_{\operatorname{large} r}=\frac{\mathrm{i} n}{8 \pi} \int_{\text {large } r} r^{2 n+1} \exp \left(-r^{2} / 4 \hat{l}^{2}-\mathrm{i} n t / 2 \pi r^{2}\right) \mathrm{d} r,
$$

where $\hat{l}^{-2} \equiv 1+l^{-2}$ and the approximation $\alpha(r) \sim 1 / 2 \pi r^{2}$ for large $r$ has been used. The argument of the exponential is stationary when

$$
r=\mathrm{i}^{1 / 4} \rho, \quad \rho=\left(2 n \hat{l}^{2} t / \pi\right)^{1 / 4}
$$

and if the contour of integration is distorted to go through the point with $\mathrm{i}^{1 / 4}=\mathrm{e}^{\mathrm{i} \pi / 8}$ the contribution from large- $r$ values is evaluated by the method of steepest descents as

$$
(f, \sigma)_{\text {large } r}=(\mathrm{in} / 8 \pi) \pi^{1 / 2} \rho^{2 n+1} \hat{l} \exp \left(-\rho^{2}(1+\mathrm{i}) / 2 \sqrt{2} \hat{l}^{2}+\mathrm{i} \pi(2 n+1) / 8\right) .
$$

We remark that this calculation of the transient relies heavily on the explicit form of the flow, the initial scalar distribution and the test function. In particular the result lacks the general applicability of the $t^{-n-1}$ contribution from the origin as given by (3.12).

In order to confirm result (4.3), figure 3(c) shows a plot of $\log |\operatorname{Re}(f, \sigma)|$ against $t / 1000$, giving phase information and good agreement between numerical values (solid) and the asymptotic formula (4.3) (dotted) within the transient regime. Note 

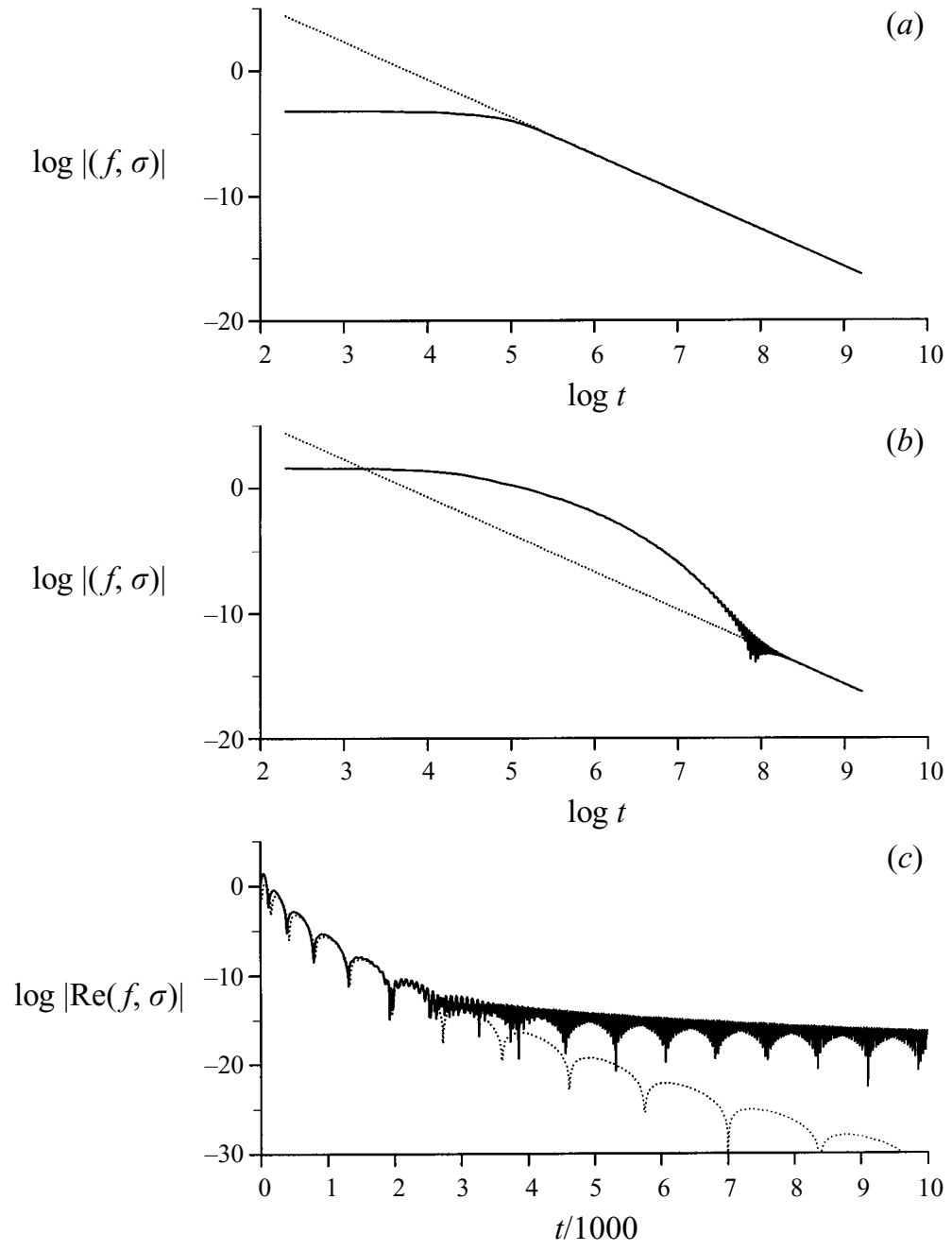

FIGURE 3. (a) $\log |(f, \sigma)|$ as a function of $\log t$ for $l=1 / 2$ and $n=2$; numerical values are solid, asymptotic values (3.12) are dotted. (b) As $(a)$ but for $l=\infty$. (c) $\log |\operatorname{Re}(f, \sigma)|$ plotted for $l=\infty$ as a function of scaled time $t / 1000$; numerical values are solid, asymptotic values (4.3) dotted.

for comparison with figure $3(b)$ that $t=3000$ in figure $3(c)$ corresponds to $\log t \simeq 8$. After the transient the $t^{-3}$ power law is recovered and the rapid oscillations show the dominance of the origin where $\sigma \propto \mathrm{e}^{-\mathrm{in} \alpha_{0} t}$. This gives a period of oscillation of $\operatorname{Re}(f, \sigma)$ of $2 \pi / n \alpha_{0}=8 \pi^{2} \simeq 78.96$ for $n=2$ in agreement with numerical results; the superposed envelope in figure $3(c)$ is a result of aliasing as the data are collected every 10 time units. Finally we note that in the latter half of our simulation scalar diffusion is beginning to play a role and plots similar to figure $2(b)$ show a damping of the rapid scalar oscillations on the $O\left(P^{1 / 3}\right)$ shear-diffuse time scale (not shown). However, the contributions to a weak measure $(f, \sigma)$ of scalar structure come mostly from regions of slow oscillation, where diffusion is least effective, and the results shown in figure 3 are insensitive to diffusion, remaining unchanged when $P$ is reduced further.

In this section we have analysed the weak convergence to zero of a passive scalar in axisymmetric flow. Under quite general assumptions an asymptotic power law of 
$t^{-n-1}$ is obtained, although there can also be a long transient arising from the far field. The transient is sensitive to the detailed structure of the vortex, perturbation and test function. In the particular case studied here, the transient decays as $\exp \left(-t^{1 / 2}\right)$ with a period of oscillation lengthening as $t^{1 / 2}$, as the contribution arises from greater radii $r \propto t^{1 / 4}$.

We have treated the case of the passive scalar in some depth since it gives clues to the vorticity problem. Recall that figure $3(b, c)$ depicts $\left(r^{n}, \sigma\right)$ while the analogous quantity $\left(r^{n}, \omega\right)$ is equal to $2 n q(t)$ by $(2.22)$, which is shown in figure $1(a, b)$. Comparison of these figures is worthwhile and it is immediately apparent that the scalar transient in figure $3(c)$ is similar to vorticity regime denoted (B) in figure $1(b)$. Particular note should be made of the way in which the frequency decreases with time in (B). We can therefore identify regime (B) as a transient contribution to $q(t)$ from the vorticity at large $r$. Regime $(\mathrm{C})$ of figure $1(a, b)$ is not dissimilar from the eventual power law of figure $3(b, c)$ and the high frequency of the oscillations suggests that it too arises from the centre $r=0$ of the vortex. However the power law is notably steeper for vorticity and clearly the coupling to the stream function is likely to be important.

\section{Vorticity asymptotics for $r=O(1)$ : the intermediate solution}

We next return to the wind-up of vorticity as governed by equations (2.4), (2.5). The purpose of this section is to develop the asymptotic solution for spiral wind-up taking $r=O(1)$ and $t \rightarrow \infty$. We should record that despite the order of presentation, the results of this section and the next were only obtained in conjunction with careful study of the numerical simulations discussed in $\S 7$.

Our starting point is the expansion in Lundgren (1982, appendix A); note that this expansion includes nonlinear terms, but we have introduced the small parameter $\varepsilon$ and will retain only linear terms (as in BL94). It is a useful first move to factor out the differential rotation in the basic flow by writing

$$
\omega=X(r, t) \exp (-\mathrm{i} n \alpha(r) t), \quad \psi=Y(r, t) \exp (-\mathrm{i} n \alpha(r) t),
$$

after which the system (2.4), (2.5) becomes (when $R e=\infty$ )

$$
\begin{gathered}
\partial_{t} X+\mathrm{i} n \beta Y=0, \\
-X=\left(-n^{2} \alpha^{\prime 2} t^{2}-\mathrm{i} n \alpha^{\prime \prime} t\right) Y-2 \mathrm{i} n \alpha^{\prime} t \partial_{r} Y-r^{-1} \mathrm{i} n \alpha^{\prime} t Y+\Delta Y .
\end{gathered}
$$

The structure of these equations suggests a large-t expansion of the type

$$
X(r, t) \sim X_{0}(r)+t^{-1} X_{1}(r)+\cdots, \quad Y(r, t) \sim t^{-2} Y_{0}(r)+t^{-3} Y_{1}(r)+\cdots
$$

under which $(5.2 a)$ becomes

and $(5.2 b)$ may be rewritten as

$$
j X_{j}=\mathrm{i} n \beta Y_{j-1}
$$

$$
Y_{j}=\left(n^{2} \alpha^{\prime 2}\right)^{-1}\left[X_{j}-\mathrm{i} n \alpha^{\prime \prime} Y_{j-1}-2 \mathrm{i} n \alpha^{\prime} Y_{j-1}^{\prime}-r^{-1} \mathrm{i} n \alpha^{\prime} Y_{j-1}+\Delta Y_{j-2}\right] .
$$

These relationships apply for all $j$ with the convention, which we shall adopt elsewhere without comment, that $X_{j}$ and $Y_{j}$ are zero for negative $j$.

This system of equations does not determine $X_{0}$ and we write $X_{0}(r)=g(r)$. The complex function $g(r)$ is determined by the initial perturbation to the vortex and its evolution through times $t=O(1)$; as a result we know very little about it. From $X_{0}$ (5.4b) determines $Y_{0}$, then (5.4a) determines $X_{1}$ and so on. This procedure thus yields 
the linearized form of Lundgren's solution

$$
\omega=\left[g(r)+\frac{\mathrm{i} \beta g(r)}{n \alpha^{\prime 2} t}+O\left(t^{-2}\right)\right] \mathrm{e}^{-\mathrm{in} \alpha(r) t}, \quad \psi=\left[\frac{g(r)}{n^{2} \alpha^{\prime 2} t^{2}}+O\left(t^{-3}\right)\right] \mathrm{e}^{-\mathrm{i} n \alpha(r) t},
$$

valid for $r=O(1)$ and $t \rightarrow \infty$. The expansion describes the decoupling of the stream function as the vorticity becomes small-scaled through differential rotation and breaks down at points where this differential rotation vanishes, $\alpha^{\prime}(r)=0$ (Lundgren 1982). In particular the appearance of the factor $\left(n^{2} \alpha^{\prime 2}\right)^{-1}$ on the right-hand side of $(5.4 b)$ shows that the series solution (5.3) is valid for $\alpha^{\prime 2} t \gg 1$ but becomes non-uniform when $\alpha^{\prime 2} t=O(1)$ : this is also apparent in (5.5a). Given the form of $\alpha(2.15)$ employed in this study the solution is non-uniform for small $r$ where $r^{2} t=O(1)$. An inner expansion is required to address this non-uniformity, and this is considered in the following section where the governing equations are rewritten in terms of $s \equiv r^{2} t$ and $t$. There is also non-uniformity at large $r$ but it turns out that it can be ignored for most purposes, and, in the interests of clarity, it is omitted from discussion in $\$ \S 5,6$. However this issue is taken up briefly in the Appendix, § A.2.

Lundgren's solution (5.5) is not the only one valid for $r=O(1)$ and $t \rightarrow \infty$. In fact the inner solution will also drive what we shall call 'Helmholtz solutions' in $r=O(1)$ : this name is chosen because of the form of $(5.9 a),(5.12 a)$ below. These are described by the expansions

$$
\begin{gathered}
\omega=X(r, t) \exp \left(-\mathrm{i} n \alpha_{0} t\right), \quad \psi=Y(r, t) \exp \left(-\mathrm{i} n \alpha_{0} t\right), \\
X(r, t) \sim t^{-\lambda}\left(X_{0}(r)+t^{-1} X_{1}(r)+\cdots\right), \quad Y(r, t) \sim t^{-\lambda}\left(Y_{0}(r)+t^{-1} Y_{1}(r)+\cdots\right) ;
\end{gathered}
$$

to avoid a plethora of symbols, we reuse $X$ and $Y$ for the various different expansions of $\omega$ and $\psi$. The exponential in (5.6) represents the rotation of the centre of the vortex, from where these solutions are driven, and the exponent $\lambda$ is to be determined. Substituting into (2.4) and (2.5) yields

$$
(-\lambda-j+1) X_{j-1}+\mathrm{i} n\left(\alpha-\alpha_{0}\right) X_{j}+\mathrm{i} n \beta Y_{j}=0, \quad-X_{j}=\Delta Y_{j}
$$

and combining the equations for $X_{0}$ and $Y_{0}$ leads to

$$
\Delta Y_{0}-\frac{\beta}{\alpha-\alpha_{0}} Y_{0}=0
$$

for the leading-order part of the solution, while higher orders are determined by

$$
\Delta Y_{j}-\frac{\beta}{\alpha-\alpha_{0}} Y_{j}=\frac{\lambda+j-1}{\mathrm{in}\left(\alpha-\alpha_{0}\right)} \Delta Y_{j-1} .
$$

As $r \rightarrow 0$ so $\alpha-\alpha_{0} \sim \alpha_{1} r^{2}$ and $\beta \sim \beta_{0}$, and equations (5.8b), (5.9a) show that solutions behave as

$$
X_{0} \sim\left(-\beta_{0} / \alpha_{1}\right) r^{ \pm w-2}, \quad Y_{0} \sim r^{ \pm w} \quad\left(w \equiv\left(n^{2}+\beta_{0} / \alpha_{1}\right)^{1 / 2}\right) . \quad(5.10 a, b, c)
$$

Note that $\beta_{0} / \alpha_{1}=8$ according to $(2.13 a)$; however we prefer not to substitute the numerical value for the present, as the algebraic form is more revealing of the structure of the problem.

For large $r$, where $\beta \simeq 0$, solutions $Y_{0}$ to $(5.9 a)$ behave as a linear combination of $r^{n}$ and $r^{-n}$ and for a physically sensible solution growth as $r \rightarrow \infty$ must be excluded. We therefore write the Helmholtz solution in the form

$$
X_{0}=-\beta h(r) /\left(\alpha-\alpha_{0}\right), \quad Y_{0}=h(r),
$$


in terms of a function $h(r)$ which satisfies

$$
\Delta h-\frac{\beta}{\alpha-\alpha_{0}} h=0
$$

subject to

$$
h(r) \sim r^{-n} \quad \text { as } \quad r \rightarrow \infty, \quad h(r) \sim h_{0} r^{-w} \quad \text { as } \quad r \rightarrow 0,
$$

in which $h_{0}$ is a constant. Boundary condition $(5.12 b)$ precludes unphysical behaviour and normalizes $h(r)$ while $(5.12 c)$ reflects the fact that at small $r, h(r)$ will contain a mixture of the two behaviours $r^{ \pm w}$ in $(5.10 b)$, of which $r^{-w}$ is the dominant. Like Lundgren's solution, this one is also non-uniform at the origin. It can be checked from (5.8), (5.9) that the leading powers in $X_{1}$ and $Y_{1}$ as $r \rightarrow 0$ are a factor of $r^{-2}$ times those in $X_{0}$ and $Y_{0}$ and so both Helmholtz and Lundgren solutions become non-uniform when $r^{2} t=O(1)$.

A combination of Lundgren's form (5.5) and a multiple $C$ of the physically acceptable Helmholtz solution (5.11) yields the complete expansion

$$
\begin{gathered}
\omega=\left[g(r)+O\left(t^{-1}\right)\right] \mathrm{e}^{-\mathrm{i} n \alpha(r) t}+C t^{-\lambda}\left[-\frac{\beta}{\alpha-\alpha_{0}} h(r)+O\left(t^{-1}\right)\right] \mathrm{e}^{-\mathrm{i} n \alpha_{0} t}, \\
\psi=\left[\frac{g(r)}{n^{2} \alpha^{\prime 2} t^{2}}+O\left(t^{-3}\right)\right] \mathrm{e}^{-\mathrm{i} n \alpha(r) t}+C t^{-\lambda}\left[h(r)+O\left(t^{-1}\right)\right] \mathrm{e}^{-\mathrm{i} n \alpha_{0} t}
\end{gathered}
$$

in $r=O(1)$ for large $t$, valid for $r^{2} t \gg 1$. For large $r, \beta(r) \rightarrow 0$ rapidly, and we expect $g(r)$ and other terms in the Lundgren solution to do the same - this is verified in the Appendix, $\S$ A.2. Thus as $r \rightarrow \infty$ so $\psi \sim C t^{-\lambda} \mathrm{e}^{-\mathrm{i} n \alpha_{0} t} r^{-n}$ and from (1.2) the identification $q(t) \sim C \mathrm{e}^{-\mathrm{in} x_{0} t} t^{-\lambda}$ may be made. The exponent $\lambda$ is indeed that describing the decay of the far field as introduced in $\S 1$.

In order to resolve the non-uniformity in solution (5.13) and to match onto the appropriate inner solution we will need to consider behaviour for small $r$ in an overlap region $t^{-1 / 2} \ll r \ll t^{-1 / 4}$. Here (5.13) is approximated as

$$
\begin{gathered}
\omega \sim\left[g_{0} r^{\mu} \mathrm{e}^{-\mathrm{i} n \alpha_{1} r^{2} t}-\frac{C h_{0} \beta_{0}}{\alpha_{1}} r^{-w-2} t^{-\lambda}\right] \mathrm{e}^{-\mathrm{i} n \alpha_{0} t}, \\
\psi \sim\left[\frac{g_{0}}{4 n^{2} \alpha_{1}^{2}} r^{\mu-2} t^{-2} \mathrm{e}^{-\mathrm{i} n \alpha_{1} r^{2} t}+C h_{0} r^{-w} t^{-\lambda}\right] \mathrm{e}^{-\mathrm{i} n \alpha_{0} t},
\end{gathered}
$$

in which it has been assumed that

$$
g(r) \sim g_{0} r^{\mu} \quad \text { as } \quad r \rightarrow 0,
$$

where $\mu$ is a power to be determined later.

In $\S 6$ we will use the variable $s=r^{2} t$ to analyse the inner region. The expressions (5.14), when cast in terms of $s$ and $t$, give that in the overlap region with $1 \ll s \ll t^{1 / 2}$,

$$
\begin{aligned}
& \omega \sim\left[g_{0} s^{\mu / 2} t^{-\mu / 2} \mathrm{e}^{-\mathrm{i} n \alpha_{1} s}-\frac{C h_{0} \beta_{0}}{\alpha_{1}} s^{-w / 2-1} t^{w / 2-\lambda+1}\right] \mathrm{e}^{-\mathrm{i} n \alpha_{0} t}, \\
& \psi \sim\left[\frac{g_{0}}{4 n^{2} \alpha_{1}^{2}} s^{\mu / 2-1} t^{-\mu / 2-1} \mathrm{e}^{-\mathrm{i} n \alpha_{1} s}+C h_{0} s^{-w / 2} t^{w / 2-\lambda}\right] \mathrm{e}^{-\mathrm{i} n \alpha_{0} t}
\end{aligned}
$$

and we recall that $w$ is given by $(5.10 c)$. The aim of analysing the inner expansion is to fix the exponents $\mu$ and $\lambda$, and also to fix $C h_{0}$ in terms of $g_{0}$. One likely condition is 
suggested immediately: since we would expect the Lundgren and Helmholtz solutions in (5.16) to have a common power of $t$ (to give greatest flexibility in the inner problem), the tentative identification

$$
2 \lambda=w+\mu+2
$$

may be made. To confirm this and in order to tie down the individual constants we next analyse the inner region.

\section{Vorticity asymptotics for $r^{2} t=O(1)$ : the inner solution}

In terms of the inner variable $s=r^{2} t$ the vorticity and stream function may be written

$$
\omega=X(s, t) \exp \left(-\mathrm{i} n \alpha_{0} t\right), \quad \psi=Y(s, t) \exp \left(-\mathrm{i} n \alpha_{0} t\right),
$$

to give from (2.4), (2.5),

$$
\partial_{t} X+s t^{-1} \partial_{s} X+\mathrm{in}\left(\alpha_{1} s t^{-1}+\alpha_{2} s^{2} t^{-2}+\cdots\right) X+\operatorname{in}\left(\beta_{0}+\beta_{1} s t^{-1}+\cdots\right) Y=0,
$$

and

$$
-t^{-1} X=4 s \partial_{s}^{2} Y+4 \partial_{s} Y-n^{2} s^{-1} Y .
$$

The structure of these partial differential equations with respect to the time variable suggests the expansions

$$
X(s, t)=t^{-\kappa}\left(X_{0}(s)+t^{-1} X_{1}(s)+\cdots\right), \quad Y(s, t)=t^{-\kappa-1}\left(Y_{0}(s)+t^{-1} Y_{1}(s)+\cdots\right), \quad(6.3 a, b)
$$

and the substitution of these into system (6.2) gives at leading order

$$
\begin{gathered}
-\kappa X_{0}+s X_{0}^{\prime}+\mathrm{i} n \alpha_{1} s X_{0}+\mathrm{i} n \beta_{0} Y_{0}=0, \\
-X_{0}=4 s Y_{0}^{\prime \prime}+4 Y_{0}^{\prime}-n^{2} s^{-1} Y_{0} .
\end{gathered}
$$

A solution of (6.4) is sought that is well-behaved at the origin in the precise sense that $\omega$ and $\psi$ must be smooth there. This means from (2.11) that a power series in $s$ for $X_{0}$ or $Y_{0}$ must begin as $s^{n / 2+j}$ where $j$ is a non-negative integer (which would usually be zero); moreover the solution must also match onto the intermediate solution (5.16) for large $s$.

It is a routine exercise to find the possible behaviours of the third-order system (6.4) for $s \gg 1$. There are three linearly independent forms:

$$
\begin{aligned}
& X_{0}=s^{\kappa} \mathrm{e}^{-\mathrm{i} n \alpha_{1} s}\left(4 n^{2} \alpha_{1}^{2}+O\left(s^{-1}\right)\right), \quad Y_{0}=s^{\kappa-1} \mathrm{e}^{-\mathrm{i} n \alpha_{1} s}\left(1+O\left(s^{-1}\right)\right), \\
& X_{0}=s^{w / 2-1}\left(-\beta_{0} / \alpha_{1}+O\left(s^{-1}\right)\right), \quad Y_{0}=s^{w / 2}\left(1+O\left(s^{-1}\right)\right), \\
& X_{0}=s^{-w / 2-1}\left(-\beta_{0} / \alpha_{1}+O\left(s^{-1}\right)\right), \quad Y_{0}=s^{-w / 2}\left(1+O\left(s^{-1}\right)\right) \text {. }
\end{aligned}
$$

A comparison with the intermediate solutions (5.16) makes it obvious that the second of these $s \gg 1$ solutions (6.6) must be rejected and this provides one constraint on the solution of the inner problem. Furthermore, if the inner solution is to match onto the Lundgren part of the intermediate forms (5.16) it is necessary that

$$
\mu=2 \kappa \text {. }
$$

Lastly, note that with the relations (5.17) and (6.8) the large-s behaviour (6.7) of the inner problem matches automatically onto the Helmholtz component of (5.16). In consequence, we expect that an appropriate inner solution of system (6.4) may 
contain parts of both (6.5) and (6.7) as $s \rightarrow \infty$, as each component can be matched satisfactorily onto the $r=O(1)$ solutions.

Next, it is necessary to consider the small-s behaviour of (6.4) in an attempt to fix the parameter $\kappa$. A solution to (6.4) of Frobenius type is based on the ansatz

$$
X_{0}=\sum_{j=0}^{\infty} x_{j} s^{\rho+j-1}, \quad Y_{0}=\sum_{j=0}^{\infty} y_{j} s^{\rho+j},
$$

in which we choose the normalization $y_{0}=1$, and yields the recurrence relations

$$
(\rho+j-1-\kappa) x_{j}+\mathrm{i} n \alpha_{1} x_{j-1}+\mathrm{i} n \beta_{0} y_{j-1}=0, \quad-x_{j}=\left[4(\rho+j)^{2}-n^{2}\right] y_{j} . \quad(6.10 a, b)
$$

Putting $j=0$ and eliminating $x_{0}$ gives the indicial equation

$$
(\rho-1-\kappa)\left(4 \rho^{2}-n^{2}\right)=0,
$$

which has roots $\rho=n / 2,-n / 2$ and $\kappa+1$. Plainly the solution corresponding to $-n / 2$ must be rejected, otherwise the smoothness requirements on the solutions at the origin would be violated.

Now suppose that $\kappa-n / 2$ is an integer. Then all three roots of the indicial equation (6.11) differ by integers and generally only one solution is well-behaved at the origin. If this one remaining solution is integrated from $s=0$ then, with $\kappa$ fixed and with no free parameters left in the problem, the large-s behaviour of system (6.4) will in general contain a mixture of all three far-field behaviours (6.5)-(6.7). This is not permitted for the reasons discussed below (6.5)-(6.7) and therefore the possibility that $\kappa-n / 2$ is an integer must in general be rejected. This turns out to be correct for azimuthal wavenumber $n \geqslant 2$ and the validity of this assumption will later be confirmed. However it turns out that $n=1$ is a very special case: for $n=1$ and $\kappa-n / 2=1$, the unique acceptable small-s solution of (6.4) turns out to have no component of (6.6) present as $s \rightarrow \infty$ and so is an acceptable solution of the inner problem. We shall postpone further discussion of the peculiarities in the $n=1$ problem until $\S 6.2$ below; until then we restrict to $n \geqslant 2$ only.

\subsection{The inner solution for $n \geqslant 2$}

The upshot of the above is that we take $n \geqslant 2$ for the moment, and $\kappa-n / 2$ not equal to an integer. This means that the $\operatorname{root} \rho=\kappa+1$ of (6.11) gives a solution for $\omega$ and $\psi$ that is not infinitely differentiable at the origin and which must therefore be discarded. All that remains is the single solution with $\rho=n / 2$ emerging from the origin, which takes the form

$$
X_{0}=s^{n / 2}\left(\mathrm{in} \beta_{0}(\kappa-n / 2)^{-1}+O(s)\right), \quad Y_{0}=s^{n / 2}(1+O(s)),
$$

and $\kappa$ is a parameter yet to be tied down.

We are left with an eigenvalue problem: system (6.4) must be solved subject to the small-s behaviour (6.12) and $\kappa$ chosen so that the large-s asymptote is a linear combination of (6.5) and (6.7); in other words, there must be no component of (6.6). This problem was tackled numerically. Accurate small- and large-s forms were calculated by generating several terms in the expansions (6.5), (6.7), (6.12). The one small-s and two large-s asymptotes were integrated numerically and matched together at a suitable point. For a given value of $\kappa$ all but one derivative could be matched automatically at this point (by appropriate choices of the coefficients of the linearly independent solutions) and iteration on $\kappa$ enabled this last derivative to be matched. 
Intriguingly, our work indicated that to within numerical accuracy

$$
\kappa=\frac{1}{2}\left(n^{2}+\beta_{0} / \alpha_{1}\right)^{1 / 2}=\frac{w}{2} .
$$

We have already mentioned that for the physical problem $n$ is an integer and $\beta_{0} / \alpha_{1}=8$ (see (2.13)), but this eigenvalue problem in an abstract sense is well-posed for any positive $n$ and $\beta_{0} / \alpha_{1}$ and the result (6.13) appeared to continue to hold even for non-physical values of these constants.

This suggested that this eigenvalue problem might be amenable to a theoretical approach and motivated a search both for analytical justification of (6.13) and for exact inner solutions. To this end, if $x_{j}$ is eliminated in favour of $y_{j}$ in (6.10) with $\rho=n / 2$ it is found that

$$
(j+1)(n+j+1)(\kappa-n / 2-j) y_{j+1}=\left(j(n+j)-\beta_{0} / 4 \alpha_{1}\right) \text { in } \alpha_{1} y_{j} .
$$

When the suggested value $\kappa=w / 2$ is used to substitute $\beta_{0} / 4=\alpha_{1}\left(\kappa^{2}-n^{2} / 4\right)$ in this recurrence relation, there is a remarkable cancellation of a (non-zero) factor $\kappa-n / 2-j$ from both sides to leave

$$
y_{j+1}=-i n \alpha_{1} y_{j} \frac{j+n / 2+\kappa}{(j+1)(j+1+n)} .
$$

This recurrence relation essentially gives coefficients of the power series expansion of Kummer's function $M(a, b, z)$. For properties of this function the reader is referred to Abramowitz \& Stegun (1965, referred to as AS65 below): we make extensive use of $\S 13$ of this book, in this case (13.1.2). Having identified the origin of the recurrence relation (6.15) the explicit solution to the eigenvalue problem may be obtained as

$$
X_{0}=\frac{\mathrm{i} n \beta_{0}}{\kappa-n / 2} s^{n / 2} M(n / 2+\kappa+1, n+1, \hat{s}), \quad Y_{0}=s^{n / 2} M(n / 2+\kappa, n+1, \hat{s}), \quad(6.16 a, b)
$$

in which $\hat{s} \equiv-\mathrm{in} \alpha_{1} s$. This solution agrees with the small-s asymptote (6.12) and it may also be checked that the solution obeys equations (6.4) directly using results (13.1.1, $4.10,4.11)$ of AS65. However, this throws little light on why this exact solution exists.

Lastly, it has to be ensured that (6.16) takes the correct asymptotic form as $s \rightarrow \infty$. In this limit, (13.5.1) of AS65 shows that

$$
X_{0} \sim \mathrm{in} \beta_{0}(\kappa-n / 2)^{-1}\left(A_{1} s^{\kappa} \mathrm{e}^{-\mathrm{i} n \alpha_{1} s}+A_{2} s^{-\kappa-1}\right), \quad Y_{0} \sim\left(A_{3} s^{\kappa-1} \mathrm{e}^{-\mathrm{i} n \alpha_{1} s}+A_{4} s^{-\kappa}\right),(6.17 a, b)
$$

with constants

$$
\begin{aligned}
A_{1} & =\left(-\mathrm{i} n \alpha_{1}\right)^{\kappa-n / 2} \frac{\Gamma(n+1)}{\Gamma(n / 2+\kappa+1)}, \quad A_{2}=\mathrm{e}^{ \pm \mathrm{i} \pi(n / 2+\kappa+1)}\left(-\mathrm{i} n \alpha_{1}\right)^{-n / 2-\kappa-1} \frac{\Gamma(n+1)}{\Gamma(n / 2-\kappa)}, \\
A_{3} & =\left(-\mathrm{i} n \alpha_{1}\right)^{\kappa-n / 2-1} \frac{\Gamma(n+1)}{\Gamma(n / 2+\kappa)}, \quad A_{4}=\mathrm{e}^{ \pm \mathrm{i} \pi(n / 2+\kappa)}\left(-\mathrm{i} n \alpha_{1}\right)^{-n / 2-\kappa} \frac{\Gamma(n+1)}{\Gamma(n / 2-\kappa+1)} .
\end{aligned}
$$

The upper sign is taken if $\alpha_{1}<0$, as for the Gaussian vortex (1.3), and the lower sign for $\alpha_{1}>0$. This solution is indeed a linear combination of the large-s asymptotic solutions (6.5), (6.7), and excludes (6.6).

The conclusion is that we have successfully constructed the inner solution explicitly and so have shown that the parameter $\kappa$ is given by equation (6.13). In deriving this solution we assumed that $\kappa-n / 2$ is not an integer. Revisiting this, we see that since $\beta_{0} / \alpha_{1}=8$ from $(2.13 a), \kappa=\left(n^{2}+8\right)^{1 / 2} / 2$ and this assumption is verified for all 
$n \geqslant 2$. However, the case $n=1$ would give $\kappa=3 / 2$, which violates the assumption, as indicated earlier; in view of the condition (2.10) of the mixing hypothesis it is perhaps unsurprising that $n=1$ is a special case, and we deal with it separately in $\S 6.2$ below.

Now that $\kappa$ is known we can identify the exponent giving the asymptotic decay rate of the far field. From (5.17), (6.8) and (6.13),

$$
\lambda=1+w=1+\left(n^{2}+\beta_{0} / \alpha_{1}\right)^{1 / 2}=1+\left(n^{2}+8\right)^{1 / 2}
$$

and this is the main result of our paper. The exponent governing the small-r dependence of $g(r)$ has the somewhat unexpected value

$$
\mu=w=\left(n^{2}+\beta_{0} / \alpha_{1}\right)^{1 / 2}=\left(n^{2}+8\right)^{1 / 2}
$$

and as a consequence for $n \geqslant 2, g(r)$ is a function which is not smooth at the origin: this lack of smoothness, of course, is dealt with by the inner solution. Comparing vorticity with a passive scalar, the coupling to the stream function leads to a greater suppression of vorticity near the origin (as $\mu>n)$; together with this comes the steeper power law $t^{-\lambda}$ as compared with $t^{-n-1}$ for a passive scalar.

Finally, we can obtain the ratio of the sizes of the Lundgren and Helmholtz solutions in the $r=O(1)$ region by requiring (6.17), (6.18) to match with (5.16). This shows that

$$
\frac{C h_{0}}{g_{0}}=-\frac{n / 2+\kappa}{\mathrm{i} n \beta_{0}} \frac{\Gamma(n / 2+\kappa)}{\Gamma(n / 2-\kappa)}\left(-\mathrm{i} n \alpha_{1}\right)^{-2 \kappa} \mathrm{e}^{\mathrm{i} \mathrm{i} \pi(n / 2+\kappa)} .
$$

\subsection{The inner solution for $n=1$}

We have already indicated that the inner problem described above requires some special care for $n=1$. In fact $n=1$ is a special case for the full linear vortex stability problem, since there is an exact time-independent solution

$$
\omega=-\mathrm{i} r \beta(r), \quad \psi=\mathrm{i} r \alpha(r)
$$

of equations (2.4), (2.5), corresponding to a translation of the basic vortex. This solution has a non-zero and constant $q(t)$. Now the mixing hypothesis applies only to initial conditions which obey (2.10) for $n=1$, and so which are orthogonal to the solution (6.20) (BL94). In fact (2.10) is equivalent to $(r, \omega)=0$ and $(r, \omega)=2 q(t)$ from (2.22), and so the condition of the mixing hypothesis amounts to $q(t)=0$ for $n=1$.

Because $n=1$ is a special case it is worth revisiting the development of $\S 5$ as well as $\S 6$. Other than noting that the exact solution (6.20) above does not fit into any of our asymptotic frameworks, the analysis of $\S 5$ goes through without modification, in particular the Lundgren and Helmholtz solutions remain valid. However, on reaching the discussion after equation (5.13), we realize that the Helmholtz solution is associated with a non-zero $q(t)$, which decays and oscillates with a frequency characteristic of the vortex centre. Since the mixing hypothesis demands $q(t)=0$, it is clear that we cannot allow the Helmholtz solution to be excited. Thus for $n=1$ only, the vorticity and stream function in the intermediate region must consist only of the Lundgren solution, that is, the multiple $C$ of the Helmholtz solution in (5.13), (5.14), (5.16) must be zero.

We move now to the inner region and take $\beta_{0} / \alpha_{1}=8$ only. Of the large-s solutions (6.5)-(6.7), we must reject not only (6.6) but also the solution (6.7) which matches to the non-existent Helmholtz solution in the intermediate region. Following the large-s asymptotic solutions, we reach the Frobenius analysis of $\S 6$. It turns out, however, that it pays to skip the derivation and move directly to assess the validity of the final 
results of this analysis. Equation (6.13) gives $\kappa=3 / 2$ and although this violates our earlier assumption that $\kappa-n / 2$ should not be an integer, the corresponding solution (6.16) is well-defined, satisfies the governing equations (6.4), and has the correct smoothness properties at the origin. Furthermore, for these values $n=1$ and $\kappa=3 / 2$ the constants $A_{2}$ and $A_{4}$ are zero (as $\Gamma(n / 2-\kappa)$ and $\Gamma(n / 2-\kappa+1)$ diverge). Thus the inner solution matches onto the Lundgren solution in the intermediate region, but contains no element of the Helmholtz solution, as required.

We therefore conclude that despite the caveats raised earlier about the case $n=1$, $\kappa=3 / 2$, the final results of the analysis (6.13), (6.16) do represent the solution of the inner problem, and the results (6.19) for the exponents remain valid in the case $n=1$. In particular $\lambda=4$ and this governs the decay of weak measures of vorticity $(f, \omega)$ as will be shown in the Appendix, $\S$ A.1 $(q(t)$ is zero and so $\lambda$ does not govern its decay). Also the Kummer functions in (6.16) simplify in this case (as does the recurrence relation (6.15)) to leave the $n=1$ inner solution as

$$
X_{0}=8 \mathrm{i} \alpha_{1} s^{1 / 2}\left(1-\mathrm{i} \alpha_{1} s / 2\right) \mathrm{e}^{-\mathrm{i} \alpha_{1} s}, \quad Y_{0}=s^{1 / 2} \mathrm{e}^{-\mathrm{i} \alpha_{1} s} .
$$

Why then do our arguments that $\kappa-n / 2$ cannot be an integer break down? Although we typically expect logarithmic terms to arise when two solutions $\rho$ of the indicial equation (here $\rho=n / 2=1 / 2, \rho=\kappa+1=5 / 2$ ) vary by an integer, this is not the case here. There is another solution of the governing equations (6.4) for $n=1$ and any $\kappa$ :

$$
X_{0}=-16 \mathrm{i} \alpha_{1} s^{1 / 2}, \quad Y_{0}=(1-2 \kappa) s^{1 / 2}+2 \mathrm{i} \alpha_{1} s^{3 / 2} .
$$

For the root $\rho=n / 2=1 / 2$ either (6.21) or (6.22) (with $\kappa=3 / 2$ ) may be taken as the Frobenius solution. For $\rho=\kappa+1=5 / 2$ a suitable linear combination of (6.21), (6.22) may be taken to eliminate terms in $s^{1 / 2}, s^{3 / 2}$ in the series for $Y_{0}$ and provide another Frobenius solution. No logarithms arise. Note that the extra solution (6.22) cannot be matched to the intermediate solution and so is absent in the physical problem, leaving only (6.21).

Although we have identified the correct $n=1$ inner solution, we mention at this stage that there is another solution that comes very close to satisfying the constraints of the inner problem. This solution has $\kappa=1$, and it was only noted because of spurious numerical results for $n=1$ discussed below in $\S 7$. When $n=\kappa=1$ one solution of (6.4) is (6.22) with $\kappa=1$ substituted, which can be written

$$
X_{0}=16 \mathrm{i} \alpha_{1} \hat{s}^{1 / 2}, \quad Y_{0}=\hat{s}^{1 / 2}(1+2 \hat{s}),
$$

with $\hat{s} \equiv-\mathrm{i} \alpha_{1} s$ as before. We can also repeat the Frobenius analysis of (6.4) along the lines described previously and these calculations show that a second solution of this system is

$$
X_{0}=\mathrm{i} \alpha_{1}\left[\left(4 \hat{s}^{3}+10 \hat{s}^{2}\right) M^{\prime}+\left(2 \hat{s}^{2}+15 \hat{s}\right) M\right], \quad Y_{0}=\hat{s}^{2} M,
$$

where $M \equiv M(1 / 2,5 / 2, \hat{s})$ and ' denotes differentiation with respect to $\hat{s}$.

Now solution (6.24) has a mixture of algebraic and oscillatory behaviour for large $s$ (cf. (6.17)), while (6.23) has purely algebraic behaviour. Remarkably it turns out that one can take a linear combination of these solutions (specifically (6.23) plus $\mp 8 \mathrm{i} / 3 \pi^{1 / 2}$ times (6.24)) to eliminate all algebraic behaviour for large $s$, leaving only a growing oscillatory solution. This far-field behaviour is exactly what is required. However this solution violates smoothness at the origin, which requires that inner solutions expand in powers $s^{1 / 2+j}$ for non-negative integers $j$ by (2.11); the Kummer part of the solution introduces integer powers of $s$. Specifically the leading term of $X_{0}$ is, correctly, $s^{1 / 2}$, 
but this is followed by a term in $s$, which is not allowed. Similarly the expansion for $Y_{0}$ begins $s^{1 / 2}, s^{3 / 2}, s^{2}$, and this $s^{2}$ term is not allowed. Thus this $n=\kappa=1$ form is not an eigensolution of system (6.4) subject to the necessary constraints. However, the loss in regularity is small compared to the leading-order terms and so it is not surprising that our numerical simulations fail to reject this 'near' solution. We will comment further on this in $\S 7$ below.

\section{Numerical study of vorticity wind-up and transient behaviour}

In order to obtain and test the results of $\S 6$, a code was written to solve the initial value problem (2.4), (2.5), (2.7), (2.9). The equations were rewritten as eight first-order partial differential equations for the real and imaginary parts of $\omega, \omega_{r}, \psi$ and $\psi_{r}$, with only those for $\omega$ containing a time derivative. This system was then integrated forward in time with a routine from the NAG suite that uses a Keller box method, together with the boundary conditions

$$
\omega=\psi=0 \quad \text { at } \quad r=0, \quad \omega=r \partial_{r} \psi+n \psi=0 \quad \text { at } \quad r=r_{\max } .
$$

The last boundary condition imposes that $\psi \sim A r^{-n}$ for large $r$. For the first set of results the parameter values

$$
n=2, \quad R e=10^{8}, \quad r_{\max }=15
$$

were used with a grid of $N=7501$ points. Large values of $r_{\max }$, certainly bigger than 10 , were needed to allow accurate evaluation of inner products (using another NAG routine), especially $\left(r^{n}, \omega\right)$.

Weak diffusion is included for numerical reasons, but has a negligible effect on the results we display. The basic axisymmetric flow (1.3) does not diffuse in our simulations, as it would only do so on an $O(R e)$ time scale, well beyond our runs (cf. BL94). The principal time scale on which diffusion acts on the perturbation is the shear-diffuse time scale of $O\left(R e^{1 / 3}\right)$. At first this appears rather short with $R e=10^{8}$; however in fact the decay is as $\exp \left(-n^{2} \alpha^{\prime 2} t^{3} / 3 R e\right)$ (Lundgren 1982; BL94) and $\alpha^{\prime}$ is numerically small (see figure $2 a$ ) with $\left|\alpha^{\prime}\right| \lesssim 0.01$. This increases the shear-diffuse time scale to about $10^{4}$. Indeed by the end of our longest runs diffusion is having some effects on the finest spiral fluctuations but not at the centre of the vortex which dominates the power law decay and the driving of the Helmholtz solution.

The main case we study is $n=2$. Figure 4(a) shows the distribution of vorticity $\omega(r)$ with real part solid, imaginary part dotted, at time $t=3000$; this may be compared with the similar figure $2(b)$ for the scalar distribution. Figure $4(b)$ shows the function $g(r)$ calculated from the vorticity distribution at $t=3000$ using $g(r) \sim \omega(r) \mathrm{e}^{\mathrm{i} n \alpha(r) t}$ for large times $t$ (see $(5.13 a)$ ). Except near the origin $g(r)$ has converged to its final form; this depends both on the initial conditions and on the evolution during $t=O(1)$, which is beyond our analysis. However, we have uncovered the small-r behaviour (5.15), $(6.19 b)$ and, in fact, $g(r)$ is approximately given by the initial condition for large $r$, as is discussed in the Appendix, $\S$ A.2.

From the results of $\S 6$ we have complete knowledge of the asymptotic form of the vorticity and stream function in the inner region $r=O\left(t^{-1 / 2}\right)$. In figure 4(c,d) we compare the numerical solution (solid) and the asymptotic solution (dotted) in this region. Specifically we set

$$
\widetilde{X}_{0}=A \mathrm{e}^{\mathrm{i} n \alpha_{0} t} t^{\kappa} s^{-n / 2} \omega, \quad \widetilde{Y}_{0}=A \mathrm{e}^{\mathrm{i} n \alpha_{0} t} t^{\kappa+1} s^{-n / 2} \psi .
$$

The right-hand side of these equations is obtained from the numerical solution at a 

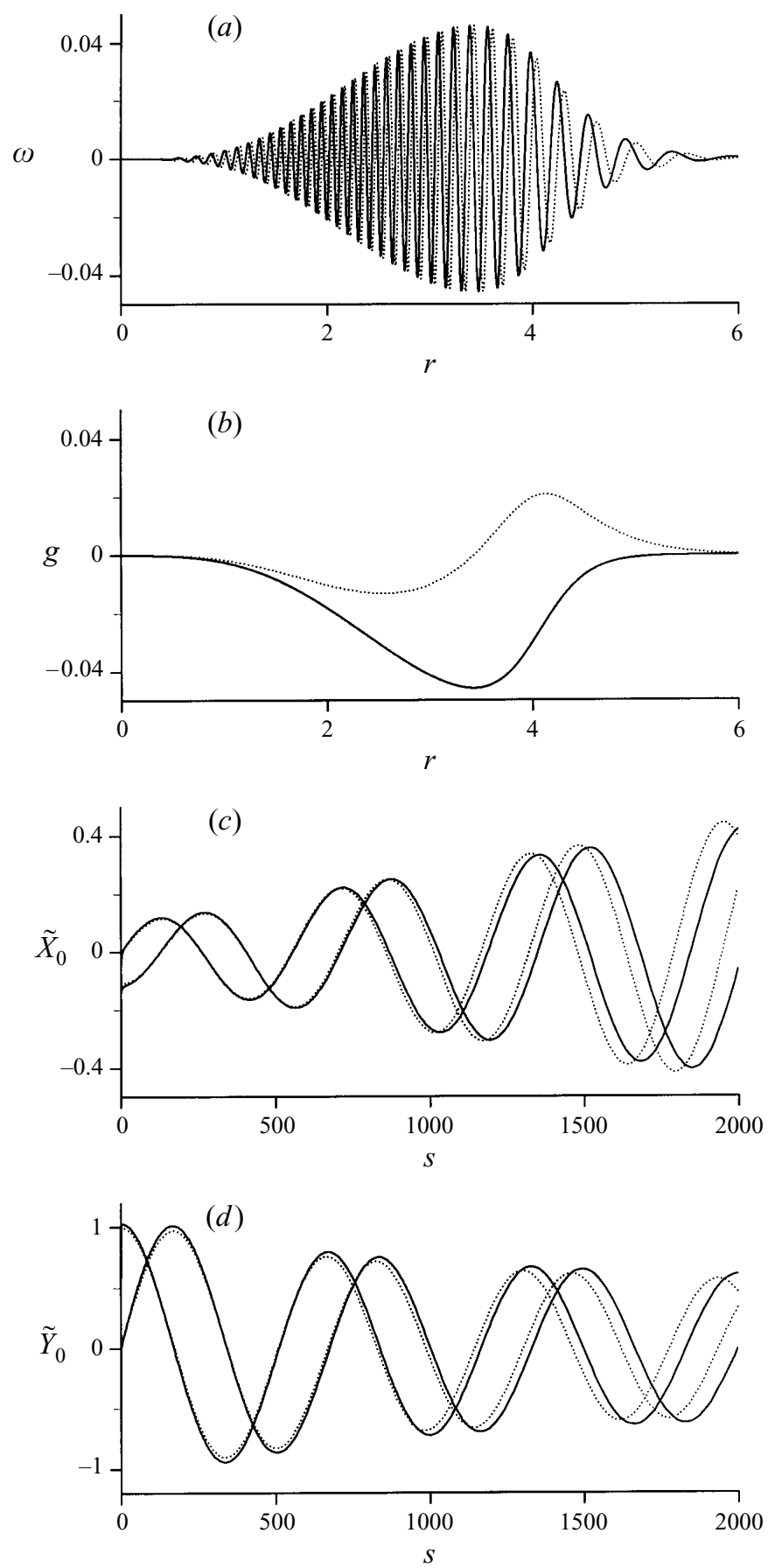

FIgURE 4. (a) Vorticity distribution $\operatorname{Re} \omega$ (solid) and $\operatorname{Im} \omega$ (dotted) against $r$, at $t=3000$ for $n=2$. (b) $g(r)$ for $n=2$ calculated from $\omega$ at $t=3000$, with real part solid, imaginary part dotted. (c) $\widetilde{X}_{0}$ plotted against $s$ for $n=2$ and $t=5000$, with numerical values solid and asymptotic values (7.4) dotted. (d) As (c) but for $\widetilde{Y}_{0}$. 

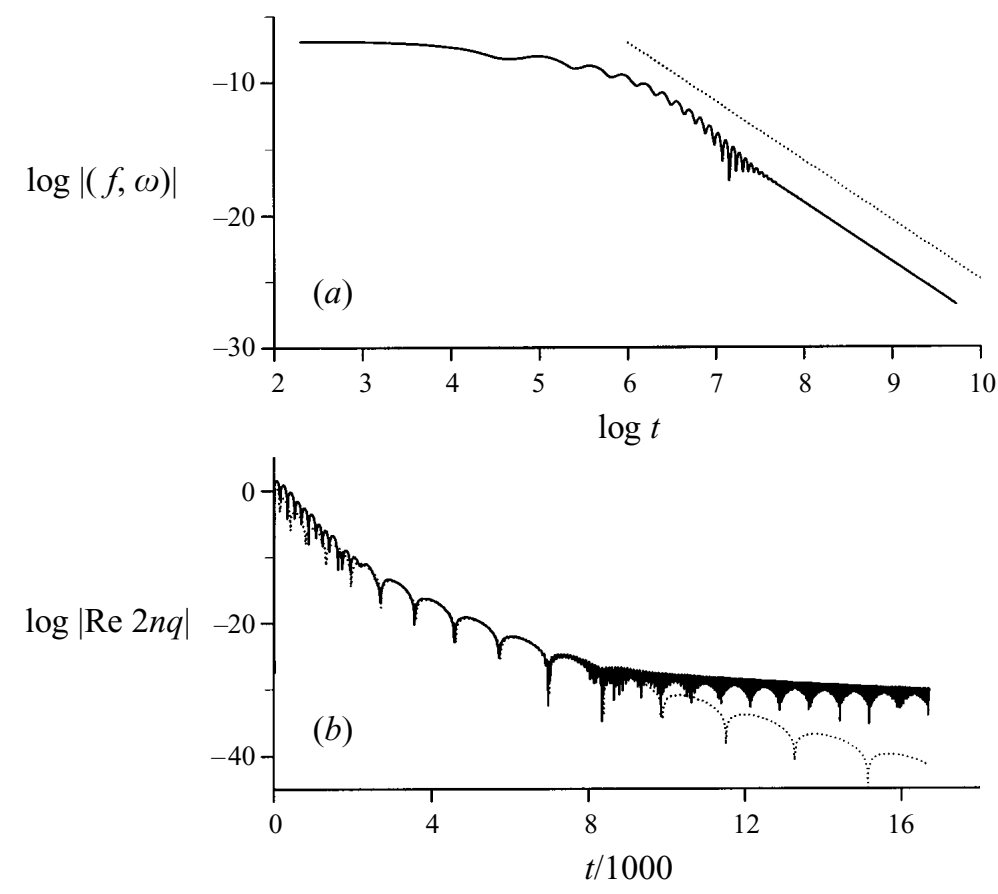

Figure 5. (a) $\log |(f, \omega)|$ (solid) as a function of $\log t$ for $l=1 / 4$ and $n=2$. The dotted line has slope -4.464 . (b) $\log |\operatorname{Re} 2 n q(t)|$ (solid) against $t / 1000$ for $l=\infty$ and $n=2$. The asymptotic approximation using (4.3) is shown dotted.

given time and the normalization constant $A$ fixed to make $\widetilde{Y}_{0}=1$ at $r=0$. From (6.2), (6.3), (6.16) the asymptotic form of these quantities, as functions of $s$ should then be

$$
\widetilde{X}_{0}=\mathrm{i} n \beta_{0}(\kappa-n / 2)^{-1} M(n / 2+\kappa+1, n+1, \hat{s}), \quad \widetilde{Y}_{0}=M(n / 2+\kappa, n+1, \hat{s}) . \quad(7.4 a, b)
$$

The Kummer functions may be evaluated directly from the series (13.1.2) in AS65. In figure $4(c)$ we show the real and imaginary parts of $\widetilde{X}_{0}$ against $s$ at $t=5000$; good agreement is observed between numerical results (solid) and the asymptotic results (dotted), and in fact the agreement improves as time increases (not shown). Figure $4(d)$ shows the same for $\widetilde{Y}_{0}$.

For $n=2$ the exponent $\lambda=1+\sqrt{12} \simeq 4.464$. This governs the final decay of $q(t)$ as seen in figure 1(a). It also gives the decay of typical weak measures of the vorticity distribution. This is because for $n \geqslant 2$ and a typical test function $f,(f, \omega)$ is dominated by the Helmholtz component of $\omega$, which decays at precisely this rate, a point justified in the Appendix, § A.1. Granted this, the $t^{-\lambda}$ power law can be seen more clearly using a test function which is localized closer to the origin. Figure 5(a) shows $\log |(f, \omega)|$ with the test function $f$ given by (2.19) with $l=1 / 2$ and decay at the correct rate is clearly seen.

Because of the global nature of the Helmholtz solution, this weak decay law will be the same for a typical test function $f$ even if it happens to decay more quickly than $O\left(r^{n}\right)$ at the origin, or even if it is zero in a neighbourhood of the origin. This may be contrasted with the situation for the passive scalar discussed at the end of $\S 4$, where the behaviour of $f$ near the origin is crucial. Note also that the behaviour of $(f, \sigma)$ also depends on the form of the scalar initial condition $\sigma\left(r, 0^{+}\right)$at the origin; 
for the vorticity problem this is unlikely to be important. For example if one took the initial condition $\omega\left(r, 0^{+}\right)$to be non-zero only in a limited range of radii, it would not remain so because of the non-local in $\beta \psi$ term in the vorticity evolution equation. One would expect that it would be hard to find initial conditions which did not lead to the generic behaviour $g(r) \sim g_{0} r^{\mu}$ with $g_{0} \neq 0$. Thus in terms of both initial conditions and test functions the $O\left(t^{-\lambda}\right)$ decay for $(f, \omega)$ has much wider applicability than the $O\left(t^{-n-1}\right)$ decay for a passive scalar.

Having confirmed the $t^{-\lambda}$ power law and the general asymptotic structure at large time $t$, we return to the transients observed for $n=2$ in figure $1(a, b)$. The transient (B) arises from passive scalar behaviour in the far-field vorticity for large $r$. In the appendix, $\S \mathrm{A} .2$ we show that the function $g(r)$ is simply given by the initial condition $g(r) \sim \omega\left(r, 0^{+}\right)$at leading order for large $r$. Thus the calculation for the passive scalar transient in $\S 4$ can be applied here to vorticity and the vorticity transient in $2 n q(t)=\left(r^{n}, \omega\right)$ is simply given by equation (4.3) with $n=2$. To check this figure $5(b)$ plots $\log |\operatorname{Re} 2 n q(t)|$ against $t / 1000$, showing this analytical approximation dotted and the numerical result (as in figure 1a) solid. Good agreement is seen during the transient (B) with the two lines being nearly indistinguishable.

The first transient (A) is characterized in figure 1(b) by an oscillation of constant period of approximately 360. As we have seen, frequency behaviour indicates the radius from which the contribution to $\left(r^{n}, \omega\right)$ and so $q(t)$ arises. In this case the oscillation corresponds to a radius of $r \simeq 4.3$. We observe that at this radius there is a peak in the imaginary part of $g(r)$ (dotted) in figure $4(b)$. The exponentially decaying transient (A) then appears to be connected with the presence of this peak. Since we have essentially no information about $g(r)$ for $r=O(1)$ beyond the numerical simulation, we are unable to describe this peak in our asymptotic framework. Furthermore the transient occurs at moderate times, probably before our long-time asymptotics becomes valid, and probably while the peak is still emerging from the initial condition. For these reasons we have no good model to account for this transient at present.

Having studied $n=2$ exhaustively we now quickly move on to other values of $n$. For $n=3, \lambda=1+\sqrt{ } 17 \simeq 5.123$. In figure $6(a)$ we confirm this value of the exponent by plotting $\log |(f, \omega)|$ (solid) with the test function (2.19) with $l=1 / 4$; decay at the correct rate (dotted) is seen clearly. Our asymptotic analysis appears good for $n=2$ and 3 and presumably continues to be so for higher values.

We have already alluded to the special nature of the $n=1$ problem, which is complicated by the mixing hypothesis. In particular the usual initial condition (2.9) violates the conditions of the hypothesis and so we do not use it. Instead we specify

$$
\omega\left(r, 0^{+}\right)=\left(r^{2}-3 \pi^{1 / 2} r / 2\right) \mathrm{e}^{-r^{2} / 4} \quad \Delta \psi\left(r, 0^{+}\right)=-\omega\left(r, 0^{+}\right)
$$

(as in BL94), which automatically satisfies the condition (2.10). The theory for $n=1$ gives $\lambda=4$ and so we expect to see an asymptotic decay of $t^{-4}$.

Figure $6(b)$ illustrates the decay of $(f, \omega)$ with $l=1 / 2$ for $n=1$. We see a clear asymptotic power law decay (solid); however the theoretical prediction shown by the dotted line with slope -4 is plainly too steep. In fact the slope is approximately 3.5 , corresponding to a value of $\kappa=1$. In this case it appears that the numerical code is picking up the spurious $n=1$ solution identified in $\S 6.2$. To confirm this, we calculate $\widetilde{X}_{0}$ and $\widetilde{Y}_{0}$ for $\kappa=1$ from (7.3); figure $6(c)$ shows comparison of the numerical values of $\widetilde{X}_{0}$ (solid) and the spurious solution (the appropriate linear combination of (6.23), (6.24)) (dotted). There is good agreement, and also for $\widetilde{Y}_{0}$ shown in figure $6(d)$. On the other hand comparison with the correct $n=1$ solution (6.21) shows complete 

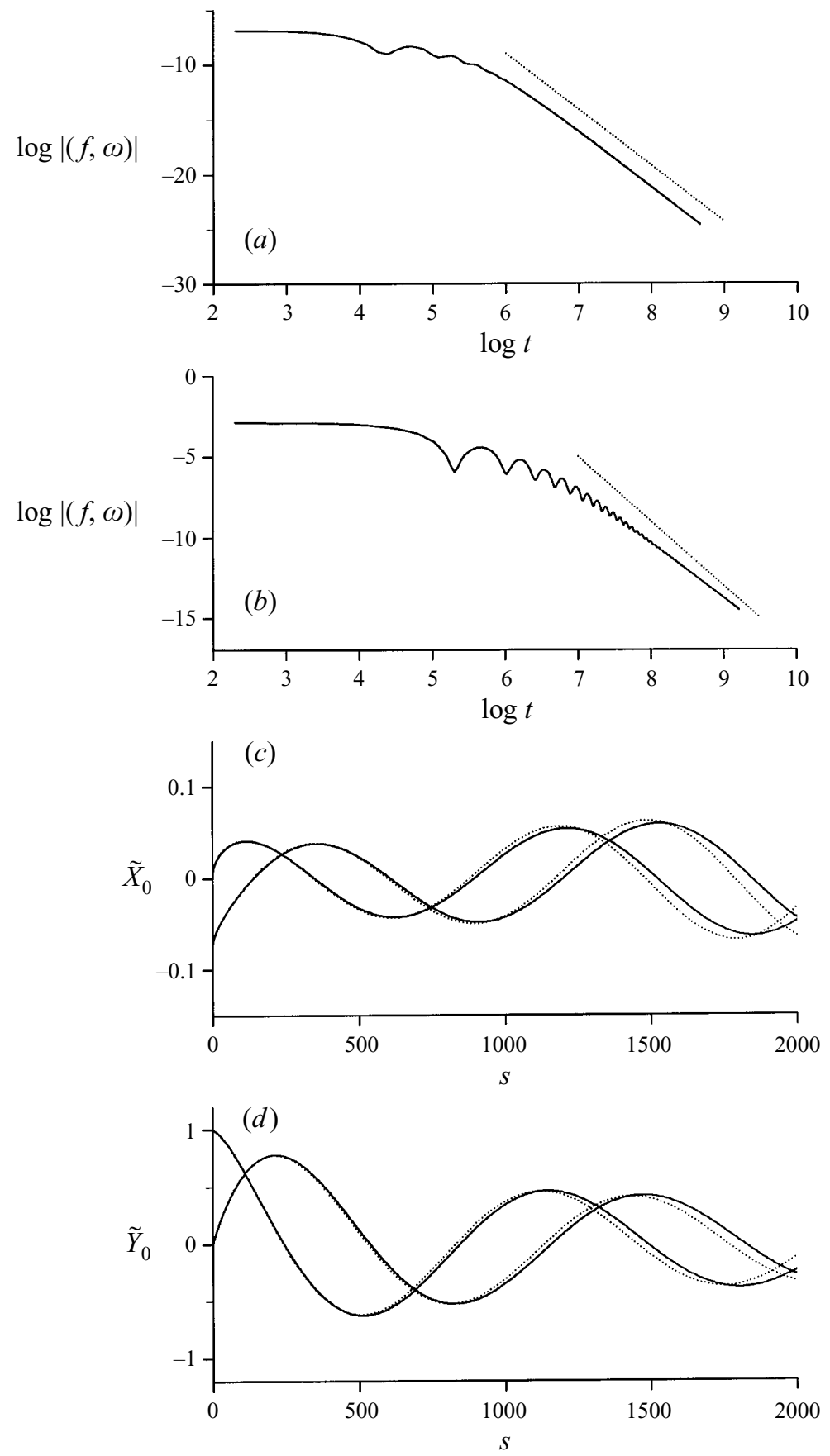

Figure 6. (a) $\log |(f, \omega)|$ (solid) as a function of $\log t$ for $l=1 / 4$ and $n=3$. The dotted line has slope -5.123. (b) As $(a)$ but for $n=1$ and initial condition (7.5). The dotted line has slope -4 . (c) $\widetilde{X}_{0}$ plotted against $s$ for $n=1$ and $t=5000$, with numerical values solid and spurious asymptotic values (obtained from (6.23) and (6.24)) dotted. $(d)$ As $(c)$ but for $\widetilde{Y}_{0}$. 
disagreement (not shown). Thus the numerical solution appears to have latched on to this incorrect solution, not surprisingly, since the solution satisfies all the constraints on the inner problem, except a weak violation of regularity at the origin.

\section{Discussion}

We have studied the relaxation of non-axisymmetric disturbances to a vortex within the linear approximation. In an inviscid framework, fluctuations of vorticity cannot be dissipated, but cascade harmlessly to small scales during the process of spiral windup. To study this relaxation to axisymmetry we look at the decay of weak measures $(f, \omega)$ of the vorticity field and a closely related quantity, the amplitude $q(t)$ of the far-field stream function. These show a universal power law, falling off as $t^{-\lambda}$ with $\lambda=1+\left(n^{2}+8\right)^{1 / 2}$. This is independent of the detailed structure of the vortex and the initial perturbation, subject only to the assumption that these are generic as detailed in $\S 2$ and the condition of the mixing hypothesis of BL94 in $\S 1$; our numerical results confirm this. For a passive scalar $\sigma$ the decay is shallower, $(f, \sigma)=O\left(t^{-n-1}\right)$. The active nature of vorticity and its coupling to the stream function appears to suppress fluctuations within the linear approximation. This occurs especially in the centre of the vortex, and is particularly striking when figures $2(b)$ and $4(a)$ are compared; recall that identical initial conditions were used for $\sigma$ and $\omega$. In fact, comparing the vertical scale, vorticity is also suppressed for $r=O(1)$. We at present lack a simple physical explanation of this process whereby vorticity is more highly suppressed than a passive scalar, and do not know whether it has applicability beyond the Gaussian vortex.

The $t^{-\lambda}$ decay can be thought of broadly as a 'phase mixing' process whereby vorticity fluctuations at different radii have different angular velocities and so become incoherent. This is a result of our assumption that the vortex has a non-trivial radial gradient of angular velocity, encapsulated by the condition $\alpha^{\prime}(r) \neq 0$ for $r>0$ which is at the heart of our analysis. Other ideas of coarse graining have been used to discuss planar vortex dynamics, in particular, averaging in a multiple scales context (e.g. Ting \& Klein 1991, §2.2) and in a statistical approach (e.g. Robert \& Sommeria 1991).

We should however note that this phase mixing effect, and the consequent weak decay is absent when an axisymmetric vortex is modelled as a circular vortex patch; this has $\omega$ constant inside a circle and zero outside, and violates condition (2.15). Perturbing the vortex patch leads in the linear approximation to waves on the vortex boundary that do not dissipate (e.g. Lamb 1932, §158; Saffman 1985), and so the function $q(t)$ oscillates but does not decay. Clearly models of vortices based on single contours are too simple to capture the internal structure of a smooth vortex, and overestimate the 'memory' of a smooth vortex to an external perturbation; similar remarks apply to truncated 'moment models' of contour dynamics (Melander, Zabusky \& Styczek 1985; Legras \& Dritschel 1991; Dritschel \& Legras 1991), which replace contours by truncated systems, typically of Hamiltonian form. Of course nonlinear effects in contour dynamics can cause waves on contours eventually to overturn and filament (Dritschel 1988b), and hence a decay of $q(t)$; however in a sense the vortex is then mimicking a smooth vorticity distribution and these are highly nonlinear effects, whereas the decay we see results from purely linear dynamics.

It is therefore important to emphasize that our results hold for vortices which are sufficiently smooth. In this context we should mentioned the role of 'stripping'; this terminology refers to the situation in which a vortex has its peripheral 'skirt' of vorticity removed by external straining (Legras \& Dritschel 1993). The resulting 
vorticity profiles have sharp edges, and so fall outside the class of smooth vortices investigated here. Recent nonlinear computations by Dritschel (1998) suggest that vortices which are far from axisymmetry and with profiles having a sharp edge tend not to axisymmetrize. Our study suggests that a worthwhile task would be to extend the present analysis to cope with profiles having steep edges. It would then be possible to examine theoretically, within linear theory, whether strong vorticity gradients can protect vortices from axisymmetrization.

Viscosity could be included in our asymptotic expansions, to make more contact with BL94 and LB95, and this remains the subject of further research. It is clear that for $R e \gg 1$ viscosity is in the nature of a fine-scale cut-off. From the work of Lundgren (1982) and BL94 it is clear that the fine spiral fluctuations decay on a $O\left(R e^{1 / 3}\right)$ time scale by the shear-diffuse mechanism. However, strictly the fluctuations decay as $\exp \left(-n^{2} \alpha^{\prime 2} t^{3} / 3 R e\right)$, and the time scale as a function of radius is $O\left(\alpha^{\prime-2 / 3} R e^{1 / 3}\right)$. This becomes non-uniform near the origin where $\alpha^{\prime}(r)=0$, and an inner solution is required here. This problem remains to be analysed. In summary, while the details of the times at which the viscous cut-off comes into operation for different parts of the vortex remain to be clarified, in particular the centre, it is apparent that our results will hold provided $1 \ll t \ll O\left(R e^{1 / 3}\right)$.

Our study has been linear and although limited in this sense, it is a necessary step in the direction of studying nonlinear problems such as the influence of spiral wind-up on resonance phenomena, vortex motion and stability (e.g. LB95; Ting \& Klein 1991). From the point of view of the full Euler equation we should also note that we have two limits $\varepsilon \rightarrow 0$ (i.e. linearization) and $t \rightarrow \infty$ : given $\varepsilon \ll 1$ do our results remain uniformly valid as $t \rightarrow \infty$ ? The answer appears to be 'yes', because as the vortex relaxes to an axisymmetric state, nonlinear effects become subdominant compared to linear terms. In fact in Lundgren's (1982) expansion nonlinear effects are included for large times $t$, but occur at higher orders of the expansion in inverse powers of $t$, beyond the linear problem we have been studying. Although further investigations here would be desirable and are underway, these results lead us to be confident that the analysis is valid for moderately small values of $\varepsilon$, uniformly in the limit $t \rightarrow \infty$. Furthermore when nonlinear effects begin to play a role, they will in the first instance lead to a modification of the dynamics for $t=O(1)$ and so only to changes in the form of the function $g(r)$.

These comments are broadly supported by nonlinear simulations of Rossi et al. (1997), who show that for moderate perturbations a Gaussian vortex relaxes to axisymmetry. However for large perturbations the vortex can bifurcate to a totally distinct stable state, in their case a tripole. Other computations in the literature have exhibited persistent non-axisymmetry (e.g. Dritschel 1989; Koumoutsakos 1997). While we expect axisymmetrization to occur in linear theory and for the nonlinear evolution of weakly perturbed vortices, these results certainly indicate that the picture can change when large perturbations are allowed.

The key to our complete asymptotic picture for large $t$ was to understand the nonuniformity of Lundgren's spiral solution at the origin. Where differential rotation is strong the Laplacian acting on a spiral distribution can be estimated as $\Delta \sim-n^{2} \alpha^{2} t^{2}$. At the origin, where differential rotation becomes small, this breaks down. We note that a similar estimation of the Laplacian is involved in the study of flux expulsion (Moffatt \& Kamkar 1983; Rhines \& Young 1983) and there is a similar non-uniformity there, which has been noted but not studied. Our basic state of an axisymmetric vortex is very simple, and it might be of interest to develop the theory for relaxation to other steady flows, for example, the strained high-Re vortices of Moffatt, Kida \& 
Ohkitani (1994) and Jiménez, Moffatt \& Vasco (1996). As a model problem one could consider switching on a persistent, rather than impulsive, external flow field. In this case the vortex remains subjected to an irrotational strain and so remains distorted while vorticity fluctuations are wound up in a flow field of elliptical streamlines. This problem is currently receiving our attention, our aim being to find out how the power law scalings of this paper are changed. More generally there is the question of how a fluid flow might relax inviscidly to a Prandtl-Batchelor state in which vorticity is uniform along streamlines (see Batchelor 1956).

The referees are thanked for their comments which led to improvements in the presentation of this work. A.D.G. gratefully acknowledges support from the Nuffield Foundation for travel and computer resources. We also thank Erik Aurell, Neil Balmforth, Nicholas Kevlahan and Konrad Bajer for valuable discussions.

\section{Appendix. Weak convergence and the outer solution}

In this appendix we tie up two loose ends from the analysis of $\S \S 5$, 6. First, we consider the evolution of weak measures $(f, \omega)$ and verify that their decay is governed by the exponent $\lambda$ obtained in $\S 6$. Secondly we consider non-uniformities in the Lundgren solution (5.5) for large $r$ and show that these are negligible for large times $t$, although they do contribute to transient behaviour.

\section{A.1. Weak convergence}

Weak measures of the vorticity distribution $\omega$ are related to those of the stream function $\psi$ by (2.21). We therefore begin by considering the evolution of $(f, \psi)$ for a test function $f$. We first restrict attention to $n \geqslant 2$ and show that the decay rate is $O\left(t^{-\lambda}\right)$.

The stream function $\psi$ is given by the Helmholtz and Lundgren solutions for $r=O(1)$ and the inner solution for $r=O\left(t^{-1 / 2}\right)$. We consider the various contributions to $(f, \psi)$ from these various pieces of $\psi$. Integrating $f$ against the Helmholtz solutions for $r=O(1)$ and $r \gg 1$ will give a contribution with a decay rate of $O\left(t^{-\lambda}\right)$ from (5.13). The remaining contributions come from the oscillatory Lundgren solution and the inner solution. From the experience gained with the scalar problem in $\S 3$, it is clear that the main contribution arises from close to the origin, in the region $r=O\left(t^{-1 / 2}\right)$ or $s=O(1)$, where the stream function $\psi=O\left(s^{n / 2} t^{-\kappa-1}\right)$ from (6.3), (6.16). Taking $f=O\left(r^{n}\right)=O\left(s^{n / 2} t^{-n / 2}\right)$ and evaluating the contribution to $(f, \psi)$ from $s=0$ to $s=O(1)$ (replacing $\left.2 r \mathrm{~d} r=t^{-1} \mathrm{~d} s\right)$ gives an estimate $(f, \psi)=O\left(t^{-n / 2-\kappa-2}\right)=$ $O\left(t^{-n / 2-w / 2-2}\right)$ (from (6.13)). Now $n / 2+w / 2+2>\lambda$ (since $\lambda=1+w$ and $w<n+2$ for $n \geqslant 2$ ) and so this represents a contribution which is steeper than $O\left(t^{-\lambda}\right)$ for $n \geqslant 2$. We therefore conclude that the exponent $\lambda$ gives the typical asymptotic decay rate of weak measures $(f, \psi)$ of the stream function and that the dominant contribution arises from the Helmholtz solution in $r=O(1)$.

For $n=1$, the Helmholtz solution is not present for $r=O(1)$, and so the dominant contribution can only come from the inner region $s=O(1)$. This contribution can be estimated from the above paragraph as again $(f, \psi)=O\left(t^{-n / 2-\kappa-2}\right)=O\left(t^{-4}\right)$ for $\kappa=3 / 2$. Now for $n=1, \lambda=4$, and so the decay rate is still $O\left(t^{-\lambda}\right)$ in this case. Thus we surmise that the appropriate decay rate for $(f, \psi)$ is given by $\lambda \equiv 1+\left(n^{2}+8\right)^{1 / 2}$ for all $n \geqslant 1$. However for $n \geqslant 2$ the largest part of $(f, \psi)$ has its origins in the Helmholtz solution which exists for $r=O(1)$, while for $n=1$ this comes from the inner zone $s=O(1)$ as the Helmholtz part is then absent. 
This completes the question of the decay of $(f, \psi)$. From identity (2.21), which is $-(f, \omega)=(\Delta f, \psi)$, it follows that weak measures $(f, \omega)$ of vorticity must decay as $t^{-\lambda}$ or steeper (to be shallower would contradict the result of the previous paragraph applied to $(\Delta f, \psi))$. In fact we observe decay at the rate $t^{-\lambda}$ in our simulations, confirming that this is a sharp estimate for the decay of typical weak measures of vorticity.

The reader may question why we took the circuitous route of dealing with $(f, \psi)$ rather than $(f, \omega)$ directly. The reason is that a naive consideration of $(f, \omega)$ can give too shallow an answer; this is because full use is not made of the fact that $\omega=-\Delta \psi$. If one straightforwardly estimates the contribution from the inner region, with $\omega=O\left(s^{n / 2} t^{-\kappa}\right)$ from (6.3), (6.16), as above, one obtains a contribution of $O\left(t^{-n / 2-w / 2-1}\right)$ to $(f, \omega)$. This is shallower than that for $(f, \psi)$ by a factor of $t$ and cannot be a sharp estimate as it would then contradict the argument of the previous paragraph (for example for $n=1$ it would give $t^{-3}$ for $(f, \omega)$ whereas we have $(f, \psi)=O\left(t^{-4}\right)$ from above) .

The resolution of this paradox is to peel off the leading $O\left(r^{n}\right)$ behaviour from $f \sim f_{0} r^{n}+O\left(r^{n+2}\right)$ and write

$$
(f, \omega)=\left(r^{n}, \omega\right) f_{0}+\left(f(r)-f_{0} r^{n}, \omega\right)=2 n q(t) f_{0}+\left(f(r)-f_{0} r^{n}, \omega\right) .
$$

The first term decays as $t^{-\lambda}$ at most, while the contribution in the inner region from $f(r)-f_{0} r^{n}=O\left(r^{n+2}\right)$ is $O\left(t^{-n / 2-w / 2-2}\right)$, as for $(f, \psi)$. In short, with these manoeuvres complete agreement is obtained between results for $(f, \psi)$ and $(f, \omega)$ for typical test functions $f$.

\section{A.2. Non-uniformity for large $r$}

Here we consider the far field $r \gg 1$. This zone is characterized primarily by the very rapid vanishing of $\beta(r)$ as our vortex is assumed localized, and the system of equations (2.4), (2.5) becomes, approximately,

$$
\partial_{t} \omega+\mathrm{in} \alpha(r) \omega=0, \quad \Delta \psi=-\omega .
$$

Driving the outer solution are the Lundgren and Helmholtz solutions for $r=O(1)$ and large $t$. It may be checked that the Helmholtz expansion (5.9) in fact remains uniform for arbitrarily large $r$, because $\beta$ vanishes rapidly, and so this solution blends seamlessly into a solution in the outer region of the form

$$
\omega_{\mathrm{H}}=0, \quad \psi_{\mathrm{H}}=q_{\mathrm{H}}(t) r^{-n} .
$$

We have established the long-time behaviour of $q_{\mathrm{H}}(t)=O\left(t^{-\lambda}\right)$ but in doing this the issue of the far-field behaviour of the Lundgren solution was ignored: in fact the Lundgren solution also gives a contribution $q_{\mathrm{L}}(t)$ to the far field with $q(t)=$ $q_{\mathrm{H}}(t)+q_{\mathrm{L}}(t)$. The role of $q_{\mathrm{L}}(t)$ was not discussed below (5.13) where we asserted that $q(t) \simeq q_{\mathrm{H}}(t)=O\left(t^{-\lambda}\right)$, in accordance with numerical simulation. However $q_{\mathrm{L}}(t)$ is certainly non-zero and so disregarding this term needs to be justified.

Related to this is the fact that the expansion (5.3) for the Lundgren solution becomes non-uniform at large $r$ for the same reason it does at small $r$ : that is the differential rotation becomes small. Inspection of (5.4) with $\beta \simeq 0$ and $\alpha \sim 1 / 2 \pi r^{2}$ for large $r$ reveals that the series becomes non-uniform when $r=O\left(t^{1 / 2}\right)$ and at these values of $r$ the estimation of $\psi$ breaks down. However $\beta$ is already exponentially small and so the stream function is effectively totally decoupled. It is clear that for large values of $r$ the Lundgren solution must match to a far-field solution of (A 1) of 
the form

$$
\omega_{\mathrm{L}}=g(r) \mathrm{e}^{-\mathrm{in} \alpha(r) t}, \quad \Delta \psi_{\mathrm{L}}=-\omega_{\mathrm{L}}
$$

and so in the Lundgren solution (5.5a) the leading term for $\omega$ remains valid for arbitrarily large $r$. On the other hand subsequent terms for $\omega$, and all terms for $\psi$ in $(5.5 b)$ are invalid for $r=O\left(t^{1 / 2}\right)$.

To this point only the smallness of $\beta(r)$ for large $r$ has been used: however $g(r)$ will also be small, as the vorticity distribution has been taken to be localized. Therefore $\omega$ is small for large $r$ and the solution (A 3) becomes

$$
\omega_{\mathrm{L}}=g(r) \mathrm{e}^{-\mathrm{i} n \alpha(r) t}, \quad \psi_{\mathrm{L}}=q_{\mathrm{L}}(t) r^{-n} .
$$

Since the leading Lundgren solution for $\omega(5.5 a)$, (A $4 a$ ) remains valid for arbitrarily large $r$, it can be used to yield information on $\psi$ even when the $\psi$ expansion has broken down. In particular it transpires that $q_{\mathrm{L}}(t)$ is dominated by the contribution from the slow oscillations in vorticity for large values of $r$.

To confirm this, let $k(r)$ be a smooth function with $k(r)=0$ for $0 \leqslant r \leqslant 1, k(r)=1$ for $r \geqslant 2$, and monotonically increasing for $1<r<2$. Define $f(r)=r^{n} k(r)$ (this is not a test function) and consider

$$
\left(f, \omega_{\mathrm{L}}\right)=-\left(\Delta f, \psi_{\mathrm{L}}\right)+2 n q_{\mathrm{L}}(t)
$$

(cf. (2.22)). Now $\Delta f=\left(k^{\prime \prime}+(2 n+1) k^{\prime} / r\right) r^{n}$ is a smooth function that vanishes outside the range $1<r<2$. In this range $\psi=O\left(t^{-2}\right)$ and oscillates at a frequency bounded away from zero; so $\left(\Delta f, \psi_{\mathrm{L}}\right)$ will vanish quickly, and generally faster than $\left(f, \omega_{\mathrm{L}}\right)$, leaving

$$
\left(f, \omega_{\mathrm{L}}\right) \simeq 2 n q_{\mathrm{L}}(t)
$$

As was seen in the example of a scalar transient of $\S 4$, the dominant contribution to such an integral comes from slow oscillations at a radius which grows with time. (The contribution from the slow oscillations at the origin is zero since $f$ is zero in $0 \leqslant r \leqslant 1$.) The decay of $\left(f, \omega_{\mathrm{L}}\right)$ and so of $q_{\mathrm{L}}(t)$ is then faster than algebraic, and so we are justified in ignoring $q_{\mathrm{L}}(t)$ in obtaining the asymptotic power law $q(t) \simeq q_{\mathrm{H}}(t)=O\left(t^{-\lambda}\right)$. Nevertheless $q_{\mathrm{L}}(t)$ can be observed as a significant transient in the manner of regime (B) of figure 1 , as we confirmed in $\S 7$.

In some cases the far-field behaviour of $g(r)$ is simply given by the initial condition $g(r)=\omega\left(r, 0^{+}\right)$and so the contribution (A 6) can be evaluated. To assess contributions to the far field, we integrate (2.4) (for $R e=\infty$ ) exactly as

$$
\omega(r, t)=\omega\left(r, 0^{+}\right) \mathrm{e}^{-\mathrm{i} n \alpha t}-\mathrm{i} n \beta \int_{0}^{t} \psi(r, \tau) \mathrm{e}^{-\mathrm{i} n \alpha(t-\tau)} \mathrm{d} \tau .
$$

In the far field $\psi \simeq q(t) r^{-n}$ with $q(t)$ decaying quickly and so the integral over time in (A 7) is $O(1)$ in time and $O\left(r^{-n}\right)$ in space. Hence

$$
\omega(r, t)=\omega\left(r, 0^{+}\right) \mathrm{e}^{-\mathrm{i} n \alpha t}+O\left(\beta r^{-n}\right) \quad \text { for } \quad r \gg 1
$$

and, provided $\omega\left(r, 0^{+}\right) \gg \beta r^{-n}$, the second term is negligible and so $g(r) \simeq \omega\left(r, 0^{+}\right)$ for large $r$ from (5.13a). This inequality is certainly satisfied for the initial condition (2.9) and then (A 6) can be evaluated explicitly. The calculation follows that of $\S 4$ concerning a passive scalar with the same initial condition and gives that $2 n q_{\mathrm{L}}(t)$ is equal to the expression in (4.1). If however $\omega\left(r, 0^{+}\right) \ll \beta r^{-n}$ then the initial condition has relatively low vorticity in the far field and this vorticity is swamped by that generated by the in $\beta \psi$ term in the governing equations. In this case we know of no simple expression for $g(r)$ as $r \rightarrow \infty$. 
REFERENCES

Abramowitz, M. \& Stegun, I. A. 1965 Handbook of Mathematical Functions. Dover (referred to herein as AS65).

BATCHELOR, G. K. 1956 A proposal concerning laminar wakes behind bluff bodies at large Reynolds number. J. Fluid Mech. 1, 388-398.

Benzi, R., Paladin, G., Patarnello, S., Santangelo, P. \& Vulpiani, A. 1986 Intermittency and coherent structures in two-dimensional turbulence. J. Phys. A: Math. Gen. 19, 3771-3784.

Bernoff, A. J. \& Lingevitch, J. F. 1994 Rapid relaxation of an axisymmetric vortex. Phys. Fluids 6, 3717-3723 (referred to herein as BL94).

Brachet, M. E., Meneguzzi, M., Politano, H. \& Sulem, P.-L. 1988 The dynamics of freely decaying two-dimensional turbulence. J. Fluid Mech. 194, 333-349.

Childress, S. \& Gilbert, A. D. 1995 Stretch, Twist, Fold: The Fast Dynamo. Lecture Notes in Physics: Monographs, vol. 37. Springer.

DrITSCHEL, D. G. 1988a Nonlinear stability bounds for inviscid, two-dimensional, parallel or circular flows with monotonic vorticity, and the analogous three-dimensional quasi-geostrophic flows. J. Fluid Mech. 191, 575-581.

DritsChel, D. G. $1988 b$ The repeated filamentation of two-dimensional vorticity interfaces. J. Fluid Mech. 194, 511-547.

DRITSCHEL, D. G. 1989 Contour dynamics and contour surgery: numerical algorithms for extended high-resolution modelling of vortex dynamics in two dimensional, inviscid, incompressible flows. Comput. Phys. Rep. 10, 77-146.

DrITSCHEL, D. G. 1998 On the persistence of non-axisymmetric vortices in inviscid two-dimensional flows. J. Fluid Mech. 371, 141-155.

Dritschel, D. G. \& Legras, B. 1991 The elliptical model of two-dimensional vortex dynamics II: disturbance equations. Phys. Fluids A 3, 855-869.

ERdÉLYI, A. 1956 Asymptotic Expansions. Dover.

Gilbert, A. D. 1988 Spiral structures and spectra in two-dimensional turbulence. J. Fluid Mech. 193, 475-497.

JimÉnez, J., Moffatt, H. K. \& VASCO, C. 1996 The structure of the vortices in freely decaying two-dimensional turbulence. J. Fluid Mech. 313, 209-222.

KerR, R. M. 1985 Higher order derivative correlations and the alignment of small-scale structures in isotropic numerical turbulence. J. Fluid Mech. 153, 31-58.

Koumoutsakos, P. 1997 Inviscid axisymmetrization of an elliptical vortex. J. Comput. Phys. 138, 821-857.

KuO, A. Y. \& CoRrsin, S. 1971 Experiments on internal intermittency and fine-structure distribution functions in fully turbulent fluid. J. Fluid Mech. 50, 285-319.

KuO, A. Y. \& Corrsin, S. 1972 Experiment on the geometry of the fine-structure regions in fully turbulent fluid. J. Fluid Mech. 56, 447-479.

Lamb, H. 1932 Hydrodynamics, 6th Edn. Cambridge University Press.

Legras, B. \& Dritschel, D. G. 1991 The elliptical model of two-dimensional vortex dynamics I: the basic state. Phys. Fluids A 3, 845-854.

LEgras, B. \& DRITSChEL, D. 1993 Vortex stripping and the generation of high vorticity gradients in two-dimensional flows. Appl. Sci. Res. 51, 445-455.

Lingevitch, J. F. \& Bernoff, A. J. 1995 Distortion and evolution of a localized vortex in an irrotational flow. Phys. Fluids 7, 1015-1026 (referred to herein as LB95).

Lundgren, T. S. 1982 Strained spiral vortex model for turbulent fine structure. Phys. Fluids 25, 2193-2203.

MCCALPIn, J. D. 1987 On the adjustment of azimuthally perturbed vortices. J. Geophys. Res. C 92, $8213-8225$.

McWilliams, J. C. 1984 The emergence of isolated coherent vortices in turbulent flow. J. Fluid Mech. 146, 21-43.

McWilliams, J. C. 1990 The vortices of two-dimensional turbulence. J. Fluid Mech. 219, 361-385.

Melander, M. V., McWilliams, J. C. \& Zabusky, N. J. 1987 Axisymmetrization and vorticitygradient intensification of an isolated two-dimensional vortex through filamentation. J. Fluid Mech. 178, 137-159.

Melander, M. V., Zabusky, N. J. \& Styczek, A. S. 1985 A moment model for vortex interactions 
of the two-dimensional Euler equations. Part 1. Computational validation of a Hamiltonian elliptical representation. J. Fluid Mech. 167, 95-115.

Moffatt, H. K. \& Kamkar, H. 1983 On the time-scale associated with flux expulsion. In: Stellar and Planetary Magnetism (ed. A. M. Soward), pp. 91-97. Gordon \& Breach.

Moffatt, H. K., KIDA, S. \& OHKITANI, K. 1994 Stretched vortices - the sinews of turbulence: large Reynolds-number asymptotics. J. Fluid Mech. 259, 241-264.

Rhines, P. B. \& Young, W. R. 1982 Homogenization of potential vorticity in planetary gyres. $J$. Fluid Mech. 122, 347-367.

Rhines, P. B. \& Young, W. R. 1983 How rapidly is a passive scalar mixed within closed streamlines? J. Fluid Mech. 133, 133-145.

Robert, R. \& Sommeria, J. 1991 Statistical equilibrium states for two-dimensional flows. J. Fluid Mech. 229, 291-310.

Rossi, L. F., Lingevitch, J. F. \& Bernoff, A. J. 1997 Quasi-steady monopole and tripole attractors for relaxing vortices. Phys. Fluids 9, 2329-2338.

Saffman, P. G. 1985 Perspectives in vortex dynamics. In Perspectives in Fluid Mechanics (ed. D. Coles), pp. 91-102. Lecture Notes in Physics, vol. 320. Springer-Verlag.

Saffman, P. G. 1992 Vortex Dynamics. Cambridge University Press.

She, Z., JACKSON, E. \& OrSZAG, S. A. 1990 Intermittent vortex structures in homogeneous isotropic turbulence. Nature 344, 226-228.

SigGiA, E. D. 1981 Numerical study of small-scale intermittency in three-dimensional turbulence. $J$. Fluid Mech. 107, 375-406.

Smith, G. B. \& Montgomery, M. T. 1995 Vortex axisymmetrization: dependence on azimuthal wave-number or asymmetric radial structure changes. Q. J. R. Met. Soc. 121, 1615-1650.

Sutyrin, G. G. 1989 Azimuthal waves and symmetrization of an intense vortex. Sov. Phys. Dokl. 34, 104-106.

Ting, L. \& Klein, R. 1991 Viscous Vortical Flows. Lecture Notes in Physics, vol. 374. Springer.

Vincent, A. \& MenEguzZI, M. 1991 The spatial structure and statistical properties of homogeneous turbulence. J. Fluid Mech. 225, 1-20.

Wan, Y. H. \& Pulvirenti, M. 1985 Nonlinear stability of circular vortex patches. Commun. Math. Phys. 99, 435-450.

YaO, H. B. \& Zabusky, N. J. 1996 Axisymmetrization of an isolated vortex region by splitting and partial merging of satellite depletion perturbations. Phys. Fluids 8, 1842-1847. 